\title{
ANALYTIC TORSION VERSUS REIDEMEISTER TORSION ON HYPERBOLIC 3-MANIFOLDS WITH CUSPS
}

\author{
JONATHAN PFAFF
}

\begin{abstract}
For a non-compact hyperbolic 3-manifold with cusps we prove an explicit formula that relates the regularized analytic torsion associated to the even symmetric powers of the standard representation of $\mathrm{SL}_{2}(\mathbb{C})$ to the corresponding Reidemeister torsion. Our proof rests on an expression of the analytic torsion in terms of special values of Ruelle zeta functions as well as on recent work of Pere Menal-Ferrer and Joan Porti.
\end{abstract}

\section{INTRODUCTION}

Let $X$ be a hyperbolic manifold with cusps of odd dimension $d$. Then $X$ is not compact but has finite volume. In a previous publication [MP2] we have introduced the analytic torsion $T_{X}(\rho)$ with coefficients in the flat vector bundle $E_{\rho}$ which is obtained by restricting a finite-dimensional complex representation $\rho$ of $G:=\operatorname{Spin}(d, 1)$ to the fundamental group $\Gamma \subset G$ of $X$. The aim of this paper is to relate the torsion $T_{X}(\rho)$ to the corresponding Reidemeister torsion invariants for the case that $X$ is 3 -dimensional.

In order to motivate our results, let us first recall the situation on a closed odd-dimensional Riemannian manifold $(M, g)$. Let $\Gamma$ denote the fundamental group of $M$ and let $\widetilde{M}$ be the universal covering space of $M$. Let $\rho$ be a finite-dimensional representation of $\Gamma$ on a complex vector space $V_{\rho}$. Moreover assume that $\rho$ is unimodular, which means that $\rho$ satisfies $|\operatorname{det} \rho(\gamma)|=1$ for all $\gamma \in \Gamma$. Let $E_{\rho}:=\widetilde{M} \times_{\rho} V_{\rho}$ be the associated flat vector bundle over $M$. Pick a Hermitian fibre metric $h$ in $E_{\rho}$. Then the analytic torsion $T_{M}(\rho) \in \mathbb{R}^{+}$is a spectral invariant of $E_{\rho}$ which depends on the metrics on $M$ and $E_{\rho}$. It is defined as a weighted product over the zeta-determinants of the Hodge-Laplace operators which act on the $E_{\rho}$-valued p-forms on $M$, see Mü2, section 2]. There exists a combinatorial counterpart of the analytic torsion, the so called Reidemeister torsion. The latter is constructed in a combinatorial way out of a smooth triangulation of $M$. It depends on a choice of bases in the homology groups $H_{*}\left(M, E_{\rho}\right)$ of $M$ with coefficients in the local system defined by $\rho$. However, via the Hodge-DeRham isomorphism and Poincaré duality, the metrics $g$ and $h$ canonically define such bases. In this way one obtains a combinatorial invariant $\tau_{M}(\rho ; h)$, the Reidemeister torsion associated to $(M, g)$ and the Hermitian vector bundle $\left(E_{\rho}, h\right)$, see Mü2, section 1, section 2]. Now the analytic torsion and the Reidemeister torsion are equal, i.e. one has $T_{M}(\rho ; h)=\tau_{M}(\rho ; h)$. For the case that $\rho$ is unitary, this was proved independently by Cheeger [Ch] and Müller [Mü1]. The extension to unimodular representations is due to Müller Mü2]. 
Let us now turn to the actual setup of this paper. We let $X$ be a hyperbolic 3-manifold which is not compact but of finite volume. If $G:=\mathrm{SL}_{2}(\mathbb{C}), K:=\mathrm{SU}(2)$, then $\widetilde{X}:=$ $G / K$ can be identified with the hyperbolic 3 -space and there exists a discrete, torsion free subgroup $\Gamma$ of $G$ such that $X=\Gamma \backslash \widetilde{X}$. One can identify $\Gamma$ with the fundamental group of $X$. Throughout this paper, we assume that $\Gamma$ satisfies a certain condition, which is formulated in equation (2.2) below. Let $\rho$ be an irreducible finite-dimensional complex representation of $G$. Restrict $\rho$ to $\Gamma$ and let $E_{\rho}$ be the associated flat vector-bundle over $X$. One can equip $E_{\rho}$ with a canonical metric, called admissible metric. The associated Laplace operator $\Delta_{p}(\rho)$ on $E_{\rho}$-valued $p$-forms has a continuous spectrum and therefore, the heat operator $\exp \left(-t \Delta_{p}(\rho)\right)$ is not trace class. So the usual zeta function regularization can not be used to define the analytic torsion. However, picking up the concept of the $b$-trace of Melrose, employed by Park in a similar context, in [MP2 we introduced the regularized trace $\operatorname{Tr}_{\text {reg }}\left(e^{-t \Delta_{p}(\rho)}\right)$ of the operators $e^{-t \Delta_{p}(\rho)}$ and in this way we extended the definition of the analytic torsion to the non-compact manifold $X$. These definitions will be reviewed in section 4 below. Let $T_{X}(\rho)$ denote the analytic torsion on $X$ associated to $\rho$.

The aim of the present article is to find a suitable generalization of the aforementioned Cheeger-Müller theorems to the specific non-compact situation of the hyperbolic 3-manifold $X$ with cusps. For $m \in \frac{1}{2} \mathbb{N}$ we let $\rho(m)$ denote the $2 m$-th symmetric power of the standard representation of $\mathrm{SL}_{2}(\mathbb{C})$. Let $\bar{X}$ be the Borel-Serre compactifcation of $X$. We recall that $\bar{X}$ is a compact smooth manifold with boundary and that $X$ is diffeomorphic to the interior of $\bar{X}$. Moreover, $\bar{X}$ and $X$ are homotopy-equivalent. Thus, every representation $\rho:=\rho(\mathrm{m})$ of $G$ also defines a flat vector bundle $\overline{E_{\rho}}$ over $\bar{X}$. Now by our assumption (2.2) on $\Gamma$ and [MePo1, Proposition 2.8], the cohomology $H^{*}(X, \rho)$ never vanishes. Thus in order to define the Reidemeister torsion of $\overline{E_{\rho}}$, one needs to fix bases in the homology $H_{*}(X, \rho)$. However, by [MP2, Lemma 7.3] the bundle $E_{\rho}$ is $L^{2}$-acyclic and thus the metrics on $X$ and $E_{\rho}$ do not give such bases. This fact is a significant difference to the situation on a closed manifold described above and causes additional difficulties. To overcome this problem, we use the normalized Reidemeister torsion which was introduced by Menal-Ferrer and Porti MePo2]. Recall that the boundary of $\bar{X}$ is a disjoint union of finitely many tori $T_{i}$. For each $i$ fix a non-trivial cycle $\theta_{i} \in H_{1}\left(T_{i} ; \mathbb{Z}\right)$. By our assumption on the group $\Gamma$, it follows from MePo2, Proposition 2.2] that the $\left\{\theta_{i}\right\}$ can be used do define a base in the homology $H_{*}(X ; \rho(m))$ for each $m \in \frac{1}{2} \mathbb{N}$. Denote the corresponding Reidemeister torsion by $\tau_{X}\left(\rho(m) ;\left\{\theta_{i}\right\}\right)$. Then by [MePo2, Proposition 2.2] for each $m \in \mathbb{N}$ the quotient

$$
\mathcal{T}_{X}(\rho(m)):=\frac{\left|\tau_{X}\left(\rho(m) ;\left\{\theta_{i}\right\}\right)\right|}{\left|\tau_{X}\left(\rho(2) ;\left\{\theta_{i}\right\}\right)\right|}
$$

is independent of the choice of the $\left\{\theta_{i}\right\}$. As explained in [MePo2, section 1, section 2], it is also independent of a given spin-structure on $X$. The number $\mathcal{T}_{X}(\rho(m))$ is called normalized Reidemeister torsion of $X$ associated to $\rho(m)$. We remark that our parametrization of the representations $\rho(m)$ differs from the one used by Menal-Ferrer and Porti in MePo2 but is consistent with the notation of [MP1], [MP2] and [Pf]. Menal-Ferrer and Porti expressed the normalized Reidemeister torsion in terms of special values of Ruelle zeta 
functions [MePo2, Theorem 5.8]. This relation generalizes a result obtained by Müller for closed hyperbolic 3-manifolds, [M̈̈2, equation 8.7, equation 8.8] and is proved via a Dehn-approximation of $X$ by closed hyperbolic 3-manfiolds. Now, in analogy to (1.1), for $m \in \mathbb{N}$ we define the normalized analytic torsion $\mathcal{T}_{X}^{\text {an }}(\rho(m))$ by

$$
\mathcal{T}_{X}^{\mathrm{an}}(\rho(m)):=\frac{T_{X}(\rho(m))}{T_{X}(\rho(2))} .
$$

Then our main result can be stated in the following theorem.

Theorem 1.1. For $m \in \mathbb{N}, m \geq 2$ we define

$$
c(m):=\frac{\prod_{j=1}^{m-1} \sqrt{(m+1)^{2}+m^{2}-j^{2}}+m}{\prod_{j=1}^{m} \sqrt{(m+1)^{2}+m^{2}-j^{2}}+m+1}\left(\frac{\sqrt{(m+1)^{2}+m^{2}}+m}{\sqrt{(m+1)^{2}+m^{2}}+m+1}\right)^{\frac{1}{2}} .
$$

Let $\kappa(X)$ be the number of cusps of $X$. Then for $m \in \mathbb{N}, m \geq 2$ one has

$$
\mathcal{T}_{X}^{\mathrm{an}}(\rho(m))=\left(\frac{c(m)}{c(2)}\right)^{\kappa(X)} \mathcal{T}_{X}(\rho(m)) .
$$

We first remark that neither the normalized Reidemeister torsion nor the quotient of the analytic torsions occuring in Theorem 1.1 are trivial. In fact, each of them is exponentially growing as $m \rightarrow \infty$. This follows for example from Theorem 1.2 below or from the more general results of MP2 . The constants $c(m)$ are a defect caused by the non-compactness of the manifold. They appear via the contribution of a certain non invariant distribution to the geometric side of the Selberg trace formula.

In a furthcoming publication, Theorem 1.1 will be applied to study for fixed $m \in \mathbb{N}$ the asymptotic behaviour of the torsion-growth in the cohomology $H^{*}\left(\Gamma_{i}, M_{\rho(m)}\right)$ for towers of arithmetic groups $\Gamma_{i} \subset \mathrm{SL}_{2}(\mathbb{C})$. Here $M_{\rho(m)}$ denotes a lattice in the representation space $V_{\rho(m)}$ of $\rho(m)$ which is stable under the $\Gamma_{i}$. In this way we will obtain a modified extension of some results of Bergeron and Venkatesh [BV] to the noncompact case. More precisely, as it was already observed by Bergeron and Venkatesh [BV, section 2] (see also [Che, equation 1.4]), the size of the torsion subgroups is closely related to the Reidemeister torsion . On the other hand, the analytic torsion $T_{X}(\rho(m))$ is accessible to compuations since it can be computed in a rather explicit form via the Selberg trace formula.

As already indicated, our proof of Theorem 1.1 is based on an expression of the analytic torsion in terms of special values of Ruelle zeta functions. Our method to establish such a relation works for every representation $\rho(m), m \in \frac{1}{2} \mathbb{Z}$. Thus for $k \in \frac{1}{2} \mathbb{N}$ we let $\sigma_{k}$ be the representation of $M:=\mathrm{SO}_{2}(\mathbb{R})$ with highest weight $k e_{2}$ as in section 2 . Then we define the Ruelle zeta function $R\left(s, \sigma_{k}\right)$ as in equation (3.4). The infinite product in (3.4) converges for $s \in \mathbb{C}$ with $\operatorname{Re}(s)>2$. We will prove the following theorem.

Theorem 1.2. Let $m \in \mathbb{N}$. Then for $m \geq 3$ one has

$$
\frac{T_{X}(\rho(m))}{T_{X}(\rho(2))}=\left(\frac{c(m)}{c(2)}\right)^{\kappa(X)} \exp \left(-\frac{1}{\pi} \operatorname{vol}(X)(m(m+1)-6)\right) \prod_{k=3}^{m}\left|R\left(k, \sigma_{k}\right)\right|
$$


where the constants $c(\rho(m))$ and $c(\rho(2))$ are as in Theorem 1.1. Similarly, for each $m \geq 1$ there exist constants $c(m+1 / 2)$, defined in (7.23) such that for $m \geq 2$ one has

$\frac{T_{X}\left(\rho\left(m+\frac{1}{2}\right)\right)}{T_{X}\left(\rho\left(\frac{3}{2}\right)\right)}=\left(\frac{c\left(m+\frac{1}{2}\right)}{c\left(\frac{3}{2}\right)}\right)^{\kappa(X)} \exp \left(-\frac{1}{\pi} \operatorname{vol}(X)(m(m+2)-3)\right) \prod_{k=2}^{m}\left|R\left(k+\frac{1}{2}, \sigma_{k+\frac{1}{2}}\right)\right|$.

If one combines the first statement of the previous Theorem with the corresponding result of Menal-Ferrer and Porti [MePo2, Theorem 5.8], Theorem 1.1 follows immediately.

We note that one can not combine Theorem 1.2 and the corresponding result MePo2, Theorem 5.8] of Menal-Ferrer and Porti to deduce an analog of Theorem 1.1 for the representations $\rho(m+1 / 2), m \in \mathbb{N}, m \geq 2$. The problem is that in this case the normalized Reidemeister torsion and the Ruelle zeta functions occuring in [MePo2, Theorem 5.8] are defined with respect to an acylic spin-structure of $X$. In the setting of the present article, this means that one replaces the group $\Gamma \subset G$ by a suitable group $\Gamma^{\prime} \subset G$ which has the same image in $\mathrm{PSL}_{2}(\mathbb{C})$ as $\Gamma$. Clearly, for each $k \in \mathbb{N}$ the Ruelle zeta functions $R\left(s, \sigma_{k}\right)$ remain the same under this change of the group $\Gamma$ since each representation $\sigma_{k}$, $k \in \mathbb{N}$ descends to a representation of $\mathrm{PSO}_{2}(\mathbb{R})$. However, this is no longer the case for the representations $\sigma_{k}, k \in \mathbb{Z}-\frac{1}{2} \mathbb{Z}$. Furthermore, Theorem 1.2 can not be applied to a group $\Gamma^{\prime}$ corresponding to an acyclic spin-structure of $X$ since by MePo1, Lemma 2.4] such a group never satisfies the assumption (2.2). This assmuption is yet needed for our compuations involving the Selberg trace formula and in order to apply the results about the meromorphic continuation of the zeta functions obtained in $[\mathrm{Pt}]$.

We shall now explain our method to prove Theorem 1.2. We first recall that on closed odd-dimensional hyperbolic manifolds Fried $[\mathrm{Fr}]$ related the behaviour of the Ruelle zeta function $R_{\rho}$ associated to a unitary representation $\rho$ of $\Gamma$ to the corresponding analytic torsion $T_{X}(\rho)$. In particular, if $\rho$ is acyclic, which means that the cohomology $H^{*}\left(X, E_{\rho}\right)$ vanishes, he showed that the function $R_{\rho}$ is regular at 0 and that $R_{\rho}(0)=T_{X}(\rho)^{2}$. However, there is no obvious method of constructing non-trivial unitary representations of (cocompact) hyperbolic lattices $\Gamma$. On the other hand, if $X$ is a closed $2 n+1$-dimensional hyperbolic manifold and if $\rho$ is a representation of $\operatorname{Spin}(2 n+1,1)$ which is not invariant under the standard Cartan-involution $\theta$, then the restriction of $\rho$ to the fundamental group $\Gamma \subset \operatorname{Spin}(2 n+1,1)$ of $X$ is an acyclic unimodular representation. Generalizing Fried's results, Bröcker [Br] and Wotzke [Wo have shown that for such representations $\rho$ the Ruelle zeta function $R_{\rho}$ is regular at 0 and that one has $R_{\rho}(0)=T_{X}(\rho)^{2}$.

We establish a generalization of the results of Bröcker and Wotzke to the non-compact hyperbolic 3-manifold $X$ which is sufficient to prove Theorem 1.2 and thereby Theorem 1.1 . The first main problem ist that the Ruelle zeta function $R_{\rho(m)}$ is a priori defined only for $s \in \mathbb{C}$ with $\operatorname{Re}(s)>2$. However, as a special case of our results obtained in $[\mathbb{P A}]$, it follows that $R_{\rho(m)}$ admits a meromorphic continuation to the entire complex plane. This is the first step in the proof. The main technical issue of this paper is now to relate the behaviour of $R_{\rho(m)}$ at zero to the regularized analytic torsion $T_{X}(\rho(m))$. Let $\rho(m)_{\theta}:=\rho(m) \circ \theta$. We prove the following proposition. 
Proposition 1.3. For $m \in \mathbb{N}$ let the constant $c(m)$ be as in Theorem 1.1. Then

$$
\begin{aligned}
& T_{X}(\rho(m))^{4} \\
= & c(m)^{4 \kappa(X)} \frac{\mathbf{C}(m: 0)}{\mathbf{C}(m+1: 0)} \lim _{s \rightarrow 0}\left(R_{\rho(m)}(s) R_{\rho(m)_{\theta}}(s) \frac{\mathbf{C}(m+1: m-s)}{\mathbf{C}(m: m+1-s)} \Gamma^{-2 \kappa(X)}(s-1)\right) .
\end{aligned}
$$

Here the functions $\mathbf{C}(k: s)$ are meromorphic functions of $s$ which are constructed out of the scattering determinant associated to the representation $\sigma$ of $M$ with highest weight ke ${ }_{2}$ and a certain $K$-type. They are defined in section 6 . For $m \in \frac{1}{2} \mathbb{N}$, a similar formula holds.

Due to the presence of the scattering term and the $\Gamma$-factor, Proposition 1.3 does not imply that the Ruelle zeta function $R_{\rho(m)}$ is regular at 0. However, from Proposition 1.3 one can deduce Theorem 1.2 which is much more explicit.

We remark that for odd-dimensional hyperbolic manifolds with cusps and for unitary representatiosn of $\Gamma$, Park studied the relation between the behaviour of the Ruelle zeta function at 0 and the analytic torsion $[\mathrm{Pa}]$. However, his results can not be applied here since the representations $\rho(m)$ are not unitary. Moreover the paper $[\mathrm{Pa}$ decisively uses the results of the earlier paper of Gon and Park [GP] on Selberg and Ruelle zeta functions and the results of this paper do not imply that the Ruelle zeta function $R_{\rho(m)}$ admits a meromorphic continuation to $\mathbb{C}$. Furthermore, in the 3-dimensional case, the paper GP only covers the Selberg and Ruelle zeta functions associated to the fundamental representations $\sigma_{0}, \sigma_{1}$ of $M$ and it is unclear whether the methods of Gon and Park can be applied to other representations of $M$ since among other things they use a special type of a Paley-Wiener theorem which presently exists only for the fundamental representations of $K$. The proof of our main results is yet based on the meromorphic continuation of the Ruelle and Selberg zeta functions associated to any representation $\sigma_{k}, k \in \mathbb{Z}$ as well as on their relation to geometric differential operators on $X$. These results have been established in our preceding paper $[\mathbb{P f}$ in the more general context of odd-dimensional hyperbolic manifolds with cusps. We want to point out that, as well as in the preceding paper $[\mathrm{Pt}]$, a lot of the methods used in the present article had been developed by Bunke and Olbrich [BO for the closed case and are generalized here to the non-compact situation. This generalization is made possible by the work of Hoffmann who proved an invariant trace formula [Ho2 and who determined the Fourier transform of the associated weighted orbital integral [Ho]].

To prove Proposition 1.3, we first express the analytic torsion $T_{X}(\rho(m))$ as a weighted product of graded determinants associated to differential operators $A(\sigma)$ for certain $\sigma \in \hat{M}$. Here the $A(\sigma)$ are of Laplace type and act on graded locally homogeneous vector bundles $E(\sigma)$ over $X$. By the same argument as in the closed case [W0], [Mü2] the product $R_{\rho(m)}(s) R_{\rho(m)_{\theta}}(s)$ can be expressed as a weighted product of Selberg zeta functions $S(s, \sigma)$ with shifted arguments for the same set of representations $\sigma \in \hat{M}$. To relate the analytic torsion to the Ruelle zeta function, we first prove a determinant formula which expresses the Selberg zeta function $S(s, \sigma)$ by the graded determinant of $A(\sigma)+s^{2}$. The prove is based on an explicit evaluation of the Laplace-Mellin transform of each term occuring on the geometric side of the Selberg trace formula applied to a particular test function $h_{t}^{\sigma}$. 
However, in contrast to the closed case, the determinant formula can only be applied to $s \in \mathbb{C}$ with $\operatorname{Re}(s)$ and $\operatorname{Re}\left(s^{2}\right)$ sufficiently large. Thus to complete the proof of Proposition 1.3, we also need to establish a functional equation for the symmetric Selberg zeta functions. Via the functional equations the scattering terms appear in Proposition 1.3.

This paper is organized as follows. In section 2 we fix notations and recall some basic facts about hyperbolic 3-manifolds. In section 3 we briefly recall the definition of the Ruelle and Selberg zeta functions. The definition of the regularized traces and the analytic torsion are reviewed in section 4 . In sections 5 and 6 we establish the determinant formula respectively the functional equations of the symmetric Selberg zeta functions. The proof of our main results is completed in the final section 7 .

Acknowledgement. This paper contains parts of the author's $\mathrm{PhD}$ thesis. He would like to thank his supervisor Prof. Werner Müller for his constant support and for helpful suggestions.

\section{Hyperbolic 3-MANifolds With CUSpS}

Let $\mathbb{H}^{3}$ denote the hyperbolic 3 -space equipped with the hyperbolic metric of constant curvature -1 . Let $G=\mathrm{SL}_{2}(\mathbb{C})$, regarded as a real Lie group, and let $K=\mathrm{SU}(2)$. Then $K$ is a maximal compact subgroup of $G$. The groups $G$ and $K$ can be identified with the groups $\operatorname{Spin}(3,1)$ and $\operatorname{Spin}(3)$ and there is a canonical isomorphism $\mathbb{H}^{3} \cong G / K$. The quotient $G / K$ will also be denoted by $\widetilde{X}$ in the sequel. Let $\mathfrak{g}$ and $\mathfrak{k}$ be the Lie algebras of $G$ and $K$. We let $\theta$ be the standard Cartan involution of $\mathfrak{g}$. The lift of $\theta$ to $G$ will be denoted by the same latter. Let $\mathfrak{g}=\mathfrak{k} \oplus \mathfrak{p}$ be the corresponding Cartan decomposition. Then the Killing form $B$ of $\mathfrak{g}$ defines an inner product on $\mathfrak{p}$. We consider the inner product $\langle\cdot, \cdot\rangle$ on $\mathfrak{p}$ which is given by $\frac{1}{4} B$. The tangent space of $\widetilde{X}$ at $1 K$ can be identified with $\mathfrak{p}$ and therefore the inner product $\langle\cdot, \cdot\rangle$ defines an invariant metric on $\tilde{X}$. This metric is the metric of constant curvature -1 .

Now we let $\Gamma$ be a discrete, torsion free subgroup of $G$ with $\operatorname{vol}(\Gamma \backslash G)<\infty$ and we let

$$
X=\Gamma \backslash \widetilde{X}
$$

We equip $X$ with the Riemannian metric induced from $\widetilde{X}$. Let $\mathfrak{P}$ be a fixed set of representatives of $\Gamma$-nonequivalent proper cuspidal parabolic subgroups of $G$. Then $\mathfrak{P}$ is finite. Throughout this paper we assume that for every $P \in \mathfrak{P}$ with Langlands decomposition $P=M_{P} A_{P} N_{P}$ one has

$$
\Gamma \cap P=\Gamma \cap N_{P}
$$

This condition is satisfied for example if $\Gamma$ is "neat", which means that the group generated by the eigenvalues of any $\gamma \in \Gamma$ contains no roots of unity $\neq 1$. It also holds for many groups $\Gamma$ which are of arithmetic significance. Let $\kappa(X):=\# \mathfrak{P}$. The geometric shape of $X$ can be described as follows, see for example [MP2]. There exists a $Y_{0}>0$ and for every $Y \geq Y_{0}$ a compact manifold $X(Y)$ with smooth boundary such that $X$ admits a 
decomposition as

$$
X=X(Y) \cup \bigsqcup_{P \in \mathfrak{P}} F_{P, Y}
$$

with $X(Y) \cap F_{P, Y}=\partial X(Y)=\partial F_{P, Y}$ and $F_{P, Y} \cap F_{P^{\prime}, Y}=\emptyset$ if $P \neq P^{\prime}$. Here the $F_{P, Y}$ are the cusps of $X$. They satisfy $F_{P, Y} \cong[Y, \infty) \times T^{2}$, where $T^{2}$ denotes the flat 2 -torus. Moreover the restriction of the metric of $X$ to $F_{P, Y}$ is given as a warped product $y^{-2} \frac{d^{2}}{d y^{2}}+y^{-2} g_{0}$, where $g_{0}$ denotes the suitably normalized standard-metric of $T^{2}$.

We let $P_{0}:=M A N$ be the standard parabolic subgroup of $G$. Then we have $M=$ $\mathrm{SO}_{2}(\mathbb{R})$. By $\mathfrak{m}, \mathfrak{a}$ and $\mathfrak{n}$ we denote the Lie algebras of $M, A$ and $N$. Then $\mathfrak{h}:=\mathfrak{a} \oplus \mathfrak{m}$ is a Cartan subalgebra of $\mathfrak{g}$ and $\mathfrak{m}$ is a Cartan subalgebra of $\mathfrak{k}$. We let $e_{1} \in \mathfrak{a}^{*}$ denote the restricted root which is implicit in the choice of $\mathfrak{n}$ and we fix $e_{2} \in i \mathfrak{m}^{*}$ such that positive roots $\Delta^{+}\left(\mathfrak{g}_{\mathbb{C}}, \mathfrak{h}_{\mathbb{C}}\right)$ can be defined by $\Delta^{+}\left(\mathfrak{g}_{\mathbb{C}}, \mathfrak{h}_{\mathbb{C}}\right):=\left\{e_{1}+e_{2}, e_{1}-e_{2}\right\}$, see [MP1, section 2]. We let $H_{1} \in \mathfrak{a}$ be such that $e_{1}\left(H_{1}\right)=1$.

By $\hat{M}$ and $\hat{K}$ we denote the equivalence classes of finite-dimensional irreducible representations of $M$ respectively $K$. For $\nu \in \hat{K}, \sigma \in \hat{M}$ we denote the multiplicity of $\sigma$ in $\left.\nu\right|_{M}$ by $[\nu: \sigma]$. Then every representation in $\hat{M}$ is one-dimensional and the elements of $\hat{M}$ will be parametrized as $\sigma_{j}, j \in \frac{1}{2} \mathbb{Z}$. Here $\sigma_{j}$ denotes the representation of $M$ with highest weight $j e_{2}$. For $l \in \frac{1}{2} \mathbb{N}$ we let $\nu_{l}$ be the representation of $K$ with highest weight $l e_{2}$. Then $\hat{K}$ is parametrized by the elements $\nu_{l}, l \in \frac{1}{2} \mathbb{N}$. Our parametrization is different from the one used in [Mü3] but consistend with the notation of [MP1], [MP2]. For $k \in \frac{1}{2} \mathbb{Z}$ we define a representation $w_{0} \sigma_{k}$ of $M$ by $w_{0} \sigma_{k}:=\sigma_{-k}$, see [MP1, section 2]. The representation rings of $M$ and $K$ will be denoted by $R(M)$ respectively $R(K)$. Then the following lemma holds.

Lemma 2.1. Let $\nu=\nu_{l}, l \in \mathbb{N}$. Then for $\sigma \in \hat{M}$ one has $[\nu: \sigma]=1$ if $\sigma=\sigma_{k}, k \in \mathbb{Z}$, $|k| \leq l$ and $[\nu: \sigma]=0$ otherwise. Let $\sigma=\sigma_{k}, k \in \mathbb{Z}-\{0\}$. For $\nu \in \hat{K}, \nu=\nu_{|k|}$ let $m_{\nu}(\sigma)=1$. For $\nu=\nu_{|k|-1}$ let $m_{\nu}(\sigma)=-1$. Finally, for $\nu \notin\left\{\nu_{|k|}, \nu_{|k|-1}\right\}$ let $m_{\nu}(\sigma)=0$. Then in $R(M)$ one has $\sigma+w_{0} \sigma=\left.\sum_{\nu \in \hat{K}} m_{\nu}(\sigma) \nu\right|_{M}$.

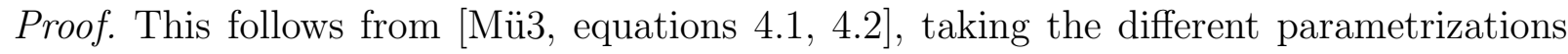
into account.

For $m \in \frac{1}{2} \mathbb{N}$ we let $\rho(m)$ denote the $2 m$-th symmetric power of the standard representation of $G=\mathrm{SL}_{2}(\mathbb{C})$ over $V_{\rho(m)}:=\mathrm{Sym}^{2 m} \mathbb{C}^{2}$. Then in the notations of [MP1], [MP2], $\rho(m)$ corresponds to the representation with highest weight $\Lambda(\rho(m)):=m e_{1}+m e_{2}$. By $\mathbb{M P 1}$, equation 2.9] we have $\rho(m) \neq \rho(m)_{\theta}$ for each $m$, where $\rho_{\theta}:=\rho \circ \theta$ for a representation $\rho$ of $G$. For $q=0,1,2$ let $\mu_{q}: M A \rightarrow \mathrm{GL}\left(\Lambda^{q} \mathfrak{n}_{\mathbb{C}}^{*}\right)$ be the $q$-th exterior power of the adjoint representation of $M A$ on $\mathfrak{n}_{\mathbb{C}}^{*}$. For $\lambda \in \mathbb{C}$ and $a \in A, a=\exp (Y), Y \in \mathfrak{a}$ we let $\xi_{\lambda}(a):=e^{\lambda e_{1}(Y)}$. Then the restriction $\left.\rho(m)\right|_{M A}$ of $\rho(m)$ to $M A$ has the following property.

Lemma 2.2. In the representation ring of $M A$ one has

$$
\left.\sum_{q=0}^{2}(-1)^{q} q \mu_{q} \otimes \rho(m)\right|_{M A}=\sigma_{m} \otimes \xi_{m+1}-\sigma_{m+1} \otimes \xi_{m}+\sigma_{-m} \otimes \xi_{-(m+1)}-\sigma_{-(m+1)} \otimes \xi_{-m} .
$$


Proof. This Lemma is a special case of [MP1, Corollary 2.6]. It can also be checked by a direct computation.

Remark 2.3. If for $k \in\{0,1\}$ the representations $\sigma_{\rho(m), k} \in \hat{M}$ and the $\lambda_{\rho(m), k} \in \mathbb{R}$ are as in [MP2, section 8], then $\sigma_{\rho(m), 0}=\sigma_{m}, \lambda_{\rho(m), 0}=m+1$ and $\sigma_{\rho(m), 1}=\sigma_{m+1}, \lambda_{\rho(m), 1}=m$.

\section{Selberg and Ruelle zeta functions}

In this section we briefly recall the definition and some properties of the Selberg and Ruelle zeta functions. For further details we refer to $[\mathbb{P A}$, section 3].

We let $\mathrm{C}(\Gamma)_{\mathrm{s}}$ denote the semisimple conjugacy classes of $\Gamma$. If $\gamma \in \Gamma$ is semisimple and nontrivial, there exists a unique $\ell(\gamma)>0$ and a $m_{\gamma} \in M$, which is unique up to conjugation in $M$, such that $\gamma$ is conjugate to $\exp \left(\ell(\gamma) H_{1}\right) m_{\gamma}$. The number $\ell(\gamma)$ is the length of the closed geodesic associated to the conjugacy class $[\gamma]$. Moreover the centralizer $Z(\gamma)$ of $\gamma$ in $\Gamma$ is an infinite cyclic group. The conjugacy class $[\gamma]$ is called prime if $\gamma$ is a generator of $Z(\gamma)$ or equivalently if the closed geodesic corresponding to $[\gamma]$ is a prime geodesic. Now for $\sigma \in \hat{M}$ the Selberg zeta function $Z(s, \sigma)$ is defined as

$$
Z(s, \sigma)=\prod_{\substack{[\gamma] \in \mathrm{C}(\Gamma)_{\mathrm{s}}-[1] \\[\gamma] \text { prime }}} \prod_{k=0}^{\infty} \operatorname{det}\left(\operatorname{Id}-\left.\sigma\left(m_{\gamma}\right) \otimes S^{k} \operatorname{Ad}\left(m_{\gamma} \exp \left(\ell(\gamma) H_{1}\right)\right)\right|_{\overline{\mathfrak{n}}} e^{-(s+n) \ell(\gamma)}\right) .
$$

By $\mathbb{P A}$, section 3] the infinite product converges for $\operatorname{Re}(s)>2$ and by $\mathbb{P} A$, Theorem 1] the function $Z(s, \sigma)$ admits a meromorphic continuation to $\mathbb{C}$.

Next for $\sigma \in \hat{M}$ we define the twisted Ruelle zeta function $R(s, \sigma)$ by

$$
R(s, \sigma):=\prod_{\substack{[\gamma] \in \mathrm{C}(\Gamma)_{s}-[1] \\[\gamma] \text { prime }}} \operatorname{det}\left(\operatorname{Id}-\sigma\left(m_{\gamma}\right) e^{-s \ell(\gamma)}\right) .
$$

The infinite product in (3.4) converges absolutely for $\operatorname{Re}(s)>2$, see $[\mathrm{Pf}$, section 3]. Furthermore, if $\rho$ is a finite-dimensional irreducible complex representation of $G$, we define the associated Ruelle zeta function $R_{\rho}(s)$ by

$$
R_{\rho}(s):=\prod_{\substack{[\gamma] \in \mathrm{C}(\Gamma)_{\mathrm{s}}-[1] \\[\gamma] \text { prime }}} \operatorname{det}\left(\mathrm{Id}-\rho(\gamma) e^{-s \ell(\gamma)}\right) .
$$

This inifinite product converges absolutely for $\operatorname{Re}(s)$ sufficiently large, see [Pf, section 3]. By $\left[\mathrm{PA}\right.$, Corollary 1.2] the functions $R_{\sigma}(s)$ and $R_{\rho}(s)$ have a meromorphic continuation to $\mathbb{C}$. We will also consider symmetric Selberg and Ruelle zeta functions. For $\sigma$ the trivial representation of $M$ we let $S(s, \sigma):=Z(s, \sigma)$ and $R_{\text {sym }}(s, \sigma):=R(s, \sigma)$. If $\sigma$ is non-trivial, we let $S(s, \sigma):=Z(s, \sigma) Z\left(s, w_{0} \sigma\right)$ and $R_{\mathrm{sym}}(s, \sigma):=R(s, \sigma) R\left(s, w_{0} \sigma\right)$.

\section{The Regularized trace And the REgularized Determinant}

In this section we define the regularized trace and the regularized analytic torsion. For further details we refer the reader to section 4 and section 5 of [MP2]. 
Let us first introduce the differential operators we consider. For a finite-dimensional unitary representation $\nu$ of $K$ over $V_{\nu}$ let $\widetilde{E}_{\nu}:=G \times_{\nu} V_{\nu}$ be the associated homogeneous vector bundle over $\widetilde{X}$. Let $E_{\nu}:=\Gamma \backslash \widetilde{E}_{\nu}$ be the corresponding locally homogeneous vector bundle over $X$. We equip $\widetilde{E}_{\nu}$ with the $G$-invariant metric induced from the metric on $V_{\nu}$. This metric pushes down to a metric on $E_{\nu}$. The smooth sections of $\widetilde{E}_{\nu}$ can be canonically identified with the space

$$
C^{\infty}(G, \nu):=\left\{f: G \rightarrow V_{\nu}: f \in C^{\infty}, f(g k)=\nu\left(k^{-1}\right) f(g), \forall g \in G, \forall k \in K\right\} .
$$

We define the space $L^{2}(G, \nu)$ in the same way. Let $\widetilde{A}_{\nu}$ be the differential operator on $\widetilde{E}_{\nu}$ which acts on $C^{\infty}(G, \nu)$ by $-\Omega$. Then by the arguments of [MP2, section 4] the operator $\widetilde{A}_{\nu}$ with domain the compactly supported functions in $C^{\infty}(G, \nu)$ is essentially selfadjoint on $L^{2}(G, \nu)$ and bounded from below. Its selfajoint closure will be denoted by $\widetilde{A}_{\nu}$ too. There exists a smooth End $\left(V_{\nu}\right)$-valued function $H_{t}^{\nu}$ which belongs to all Harish-Chandra-Schwarz spaces and which satisfies $H_{t}^{\nu}\left(k^{-1} g k^{\prime}\right)=\nu(k)^{-1} \circ H_{t}^{\nu}(g) \circ \nu\left(k^{\prime}\right)$ for all $k, k^{\prime} \in K$ an for all $g \in G$ such that $e^{-t \tilde{A}_{\nu}}$ acts on $L^{2}(G, \nu)$ as a convolution operator with kernel $H_{t}^{\nu}$, see [MP2, equation 4.7]. If $C^{\infty}(\Gamma \backslash G, \nu)$ are the $\Gamma$-invariant elements of $C^{\infty}(G, \nu)$, then the smooth sections of $E_{\nu}$ can be identified with $C^{\infty}(\Gamma \backslash G, \nu)$. Similarly, the square-integrable sections of $E_{\nu}$ can be identified with the $\Gamma$-invariant elements $L^{2}(\Gamma \backslash G, \nu)$ of $L^{2}(G, \nu)$. Let $A_{\nu}$ be the differential operator on $E_{\nu}$ which acts as $-\Omega$ on $C^{\infty}(\Gamma \backslash G, \nu)$. Then $A_{\nu}$ with domain the compactly supported elements in $C^{\infty}(\Gamma \backslash G, \nu)$ is again bounded from below and essentially selfadjoint on $L^{2}(\Gamma \backslash G, \nu)$ and its closure will be denoted by the same symbol. Let $\lambda_{\nu, 0} \leq \lambda_{\nu, 1} \leq \ldots$ be the sequence of eigenvalues of $A_{\nu}$, counted with multiplicity. One can easily extend Theorem I.1 of [DO] and its proof to the operators $A_{\nu}$ and thus there exists a constant $C>0$ such that for each $\lambda>0$ one has

$$
\#\left\{j: \lambda_{\nu, j} \leq \lambda\right\} \leq C(1+\lambda)^{\frac{3}{2}} .
$$

Now consider the heat-semigroup $e^{-t A_{\nu}}$ of $A_{\nu}$ acting on $L^{2}(\Gamma \backslash G, \nu)$. The operator $e^{-t A_{\nu}}$ is an integral operator on $L^{2}(\Gamma \backslash G, \nu)$ with smooth kernel $H^{\nu}\left(t ; x, x^{\prime}\right)$ defined in MP2, equation 4.8]. Let $h^{\nu}\left(t ; x, x^{\prime}\right):=\operatorname{Tr} H^{\nu}\left(t ; x, x^{\prime}\right)$. The operator $e^{-t A_{\nu}}$ is not trace class and $h^{\nu}(t ; x, x)$ is not integrable over $X$. However, it follows from the Mass-Selberg relations, that with respect to the decomposition (2.3) the integral of $h^{\nu}(t ; x, x)$ over $X(Y)$ has an asymptotic expansion in $Y$ as $Y \rightarrow \infty$ and, following ideas of Melrose, one can take the finite part in this expansion as a definition of the regularized trace $\operatorname{Tr}_{\text {reg }}\left(e^{-t A_{\nu}}\right)$ of $e^{-t A_{\nu}}$. Explicitly, one obtains

$$
\begin{aligned}
\operatorname{Tr}_{\mathrm{reg}}\left(e^{-t A_{\nu}}\right) & =\sum_{j} e^{-t \lambda_{\nu, j}}+\sum_{\substack{\sigma \in \hat{M} ; \sigma=w_{0} \sigma \\
[\nu: \sigma] \neq 0}} e^{t c(\sigma)} \frac{\operatorname{Tr}(\widetilde{\boldsymbol{C}}(\sigma, \nu, 0))}{4} \\
& -\frac{1}{4 \pi} \sum_{\substack{\sigma \in \hat{M} \\
\nu: \sigma] \neq 0}} \int_{\mathbb{R}} e^{-t\left(\lambda^{2}-c(\sigma)\right)} \operatorname{Tr}\left(\widetilde{\boldsymbol{C}}(\sigma, \nu,-i \lambda) \frac{d}{d z} \widetilde{\boldsymbol{C}}(\sigma, \nu, i \lambda)\right) d \lambda,
\end{aligned}
$$


see [MP2, equation 5.2, definition 5.1]. Here the first sum on the right hand side of (4.7) converges absolutely by (4.6) and all integrals converge absolutely by the arguments of [MP2, section 5]. The functions $\widetilde{\boldsymbol{C}}(\sigma, \nu, z)$ are meromorphic functions of $z$ with values in the endomorphisms of a finite-dimensional vector-space, which are regular and invertible on $i \mathbb{R}$. They are constructed out of the constant term-matrices, also called scattering matrices, associated to the Eisenstein series, see [MP2, section 3, section 5]. The constansts $c(\sigma)$ are defined by $c\left(\sigma_{j}\right):=j^{2}-1, j \in \frac{1}{2} \mathbb{Z}$.

The key fact which makes the regularized trace accessible to computations is that the right hand side of (4.7) equals the spectral side of the Selberg trace formula applied to the function $h_{t}^{\nu}:=\operatorname{Tr} H_{t}^{\nu}$. The spectral side of the trace formula consists of a sum of tempered distributions. We shall now define these distributions in the form in which they will be used for the subsequent computations. For further details we refer to [MP2, section 6]. If $\sigma \in \hat{M}, \lambda \in \mathbb{C}$, we let $\pi_{\sigma, \lambda}$ be the principle-series representation of $G$ as in MP2, section 2.7]. Then $\pi_{\sigma, \lambda}$ is unitary iff $\lambda$ is real. The global character of $\pi_{\sigma, \lambda}$ will be denoted by $\Theta_{\sigma, \lambda}$. Let $\alpha$ be a $K$-finite Schwarz function. The identity and the hyperbolic term are defined by

$$
I(\alpha):=\operatorname{vol}(X) \sum_{\sigma \in \hat{M}} \int_{\mathbb{R}} \Theta_{\sigma, \lambda}(\alpha) P_{\sigma}(i \lambda) d \lambda ; \quad H(\alpha):=\int_{\Gamma \backslash G} \sum_{\gamma \in \Gamma_{s}-1} \alpha\left(x^{-1} \gamma x\right) d x .
$$

Here $P_{\sigma}$ is the Plancherel polynomial. Explicitly, for $k \in \frac{1}{2} \mathbb{Z}$ one has

$$
P_{\sigma_{k}}(z)=\frac{1}{4 \pi^{2}}\left(k^{2}-z^{2}\right)
$$

see [MP1], Mü3]. Moreover, $\Gamma_{\mathrm{s}}$ are the semisimple elements of $\Gamma$. Next for each $\sigma$ in $\hat{M}$ we define a meromorphic function $\Omega(\sigma, \lambda)$ as in [MP2, Theorem 6.2] and we define the constant $C(\Gamma)$ as in MP2, page 22]. Then the distributions $\mathcal{I}$ and $T$ are defined as

$$
\mathcal{I}(\alpha):=\frac{\kappa(X)}{4 \pi} \sum_{\sigma \in \hat{M}} \int_{\mathbb{R}} \Theta_{\sigma, \lambda}(\alpha) \Omega(\check{\sigma},-\lambda) d \lambda ; \quad T(\alpha):=\frac{C(\Gamma)}{2 \pi} \sum_{\sigma \in \hat{M}} \int_{\mathbb{R}} \Theta_{\sigma, \lambda}(\alpha) d \lambda
$$

Finally let $J_{P_{0} \mid \bar{P}_{0}}(\sigma, z)$ be the Knapp-Stein intertwining operator defined as in MP2, equation 6.6]. Then $J_{P_{0} \mid \bar{P}_{0}}(\sigma, z)$ is a meromorphic function of $z \in \mathbb{C}$ which is regular and invertible on $\mathbb{R}-\{0\}$. Let $H_{\epsilon}$ be the half-circle from $-\epsilon$ to $\epsilon$ in the lower half-plane, oriented counter-clockwise. Let $D_{\epsilon}$ be the path which is the union of $(-\infty,-\epsilon], H_{\epsilon}$ and $[\epsilon, \infty)$. Then the distribution $J$ is defined by

$$
J(\alpha):=-\sum_{\sigma \in \hat{M}} \frac{\kappa(X)}{4 \pi i} \int_{D_{\epsilon}} \operatorname{Tr}\left(J_{\bar{P}_{0} \mid P_{0}}(\sigma, \zeta)^{-1} \frac{d}{d \zeta} J_{\bar{P}_{0} \mid P_{0}}(\sigma, \zeta) \pi_{\sigma, \zeta}(\alpha)\right) d \zeta .
$$

By the Selberg trace formula, one can express the regularized trace as

$$
\operatorname{Tr}_{\mathrm{reg}}\left(e^{-t A_{\nu}}\right)=I\left(h_{t}^{\nu}\right)+H\left(h_{t}^{\nu}\right)+T\left(h_{t}^{\nu}\right)+\mathcal{I}\left(h_{t}^{\nu}\right)+J\left(h_{t}^{\nu}\right),
$$

see [MP2, Theorem 6.1, Theorem 6.2]. 
Next we introduce the spectral zeta function associated to $A_{\nu}+s$ for certain $s \in \mathbb{C}$. If $\lambda_{\nu} \in \mathbb{R}$ is the smallest eigenvalue of $A_{\nu}$, we define $b(\nu) \in \mathbb{R}$ by

$$
b(\nu):=\max \left\{\{c(\sigma): \sigma \in \hat{M}:[\nu: \sigma] \neq 0\} \sqcup\left\{-\lambda_{\nu}\right\}\right\},
$$

where the constants $c(\sigma)$ are as above.

Proposition 4.1. Let $s \in \mathbb{C}$ with $\operatorname{Re}(s)>b(\nu)$. Then for $\operatorname{Re}(z)>\frac{d}{2}$ the integral

$$
\xi_{\nu}(s, z):=\int_{0}^{\infty} t^{z-1} \operatorname{Tr}_{\mathrm{reg}}\left(e^{-t\left(A_{\nu}+s\right)}\right) d t
$$

converges and $\xi_{\nu}$ is holomorphic on $\left\{(s, z) \in \mathbb{C} \times \mathbb{C}: \operatorname{Re}(s)>b(\nu): \operatorname{Re}(z)>\frac{d}{2}\right\}$. Moreover, $\xi_{\nu}(s, z)$ has a continuation to a holomorphic function on $\{(s, z) \in \mathbb{C} \times \mathbb{C}: \operatorname{Re}(s)>b(\nu): z \neq$ $\left.-j, z \neq 3 / 2-j, j \in \mathbb{N}_{0}\right\}$. For every $s \in \mathbb{C}$ with $\operatorname{Re}(s)>b(\nu)$ the function $z \mapsto \xi_{\nu}(s, z)$ is a meromorphic function on $\mathbb{C}$ with an at most simple pole at $z=0$ and its residue at $z=0$ is independent of $s$.

Proof. By (4.7) there exists a constant $C$ such that $\left|\operatorname{Tr}_{\mathrm{reg}} e^{-t\left(A_{\nu}+s\right)}\right| \leq C e^{-t(\operatorname{Re}(s)-b(\nu))}$. Thus the integral $\int_{1}^{\infty} t^{z-1} \operatorname{Tr}_{\mathrm{reg}} e^{-t\left(A_{\nu}+s\right)}$ converges absolutely for all $\{(z, s) \in \mathbb{C} \times \mathbb{C}: \operatorname{Re}(s)>$ $b(\nu)\}$ and is holomorphic there. Expanding $e^{-t s}$ in a power series, it follows from MP2, Proposition 6.9] that one has an asymptotic expansion

$$
\operatorname{Tr}_{\mathrm{reg}} e^{-t\left(A_{\nu}+s\right)} \sim \sum_{j=0}^{\infty} a_{j}(s) t^{j-\frac{3}{2}}+\sum_{j=0}^{\infty} b_{j}(s) t^{j-\frac{1}{2}} \log t+\sum_{j=0}^{\infty} c_{j}(s) t^{j}
$$

as $t \rightarrow+0$ which holds locally uniformly in $s$. Here the coefficients $a_{j}(s), b_{j}(s)$ and $c_{j}(s)$ depend holomorphically on $s$ and by [MP2, Proposition 6.9] and the fact that $d=3$ is odd it follows that $c_{0}(s)$ is independent of $s$. Thus the Proposition follows from standard methods which are described for example in [Gi].

Now we can define the regularized determinant proceeding as on a closed manifold. By Proposition 4.1, for $s \in \mathbb{C}$ with $\operatorname{Re}(s)>b(\nu)$ the function $\xi_{\nu}(s, z) / \Gamma(z)$ is regular at $z=0$. Thus for $s \in \mathbb{C}$ with $\operatorname{Re}(s)>b(\nu)$ we define the determinant of $A_{\nu}+s$ by

$$
\operatorname{det}\left(A_{\nu}+s\right):=\exp \left(-\left.\frac{\partial}{\partial z}\right|_{z=0} \frac{\xi_{\nu}(s, z)}{\Gamma(z)}\right) .
$$

This definition generalizes the definition of the zeta-regularized determinant of a positive elliptic differential operator on a closed manifold. We remark that one can easily show that $-b(\nu)$ equals the infimum of the spectrum of $A_{\nu}$. This fact puts the definition of $b(\nu)$ into a natural context. However it will not be used here.

We finally turn to the definition of the analytic torsion. For further details we refer to [MP2, section 7]. Let $\rho$ be a finite-dimensional irreducible complex representation of $G$ which is not invariant under $\theta$. Let $E_{\rho}^{\prime}$ be the flat vector bundle associated to the restriction of $\rho$ to $\Gamma$. Then $E_{\rho}^{\prime}$ is canonically isomorphic to the locally homogeneous vector bundle $E_{\rho}$ associated to $\left.\rho\right|_{K}$. For $p=0, \ldots, 3$ we define $\nu_{p}(\rho):=\Lambda^{p} \operatorname{Ad}^{*} \otimes \rho: K \rightarrow \operatorname{GL}\left(\Lambda^{p} \mathfrak{p}^{*} \otimes V_{\rho}\right)$. There is a canonical isomorphism $\Lambda^{p}\left(E_{\rho}\right) \cong \Gamma \backslash\left(G \times_{\nu_{p}(\rho)}\left(\Lambda^{p} \mathfrak{p}^{*} \otimes V_{\rho}\right)\right)$. By MtM, Lemma 
3.1], the bundle $E_{\rho}$ carries a canocial invariant metric, called admissible metric and if $\Delta_{p}(\rho)$ denotes the corresponding flat Hodge-Laplace operator acting on the $E_{\rho}$-valued $p$-forms, then by Kuga's formula $\Delta_{p}(\rho)$ acts on $C^{\infty}\left(\Gamma \backslash G, \nu_{p}(\rho)\right)$ as $-\Omega+\rho(\Omega)$, see [MtM, equation 6.9]. By [MP2, Lemma $7.1(2)$, Lemma 7.3], for $p=0, \ldots, 3$ one has $\rho(\Omega)-b_{\nu_{p}(\rho)} \geq \frac{1}{4}$, where the $b_{\nu_{p}(\rho)}$ are as in (4.11). Thus the determinants $\operatorname{det}\left(\Delta_{p}(\rho)\right):=\operatorname{det}\left(A_{\nu_{p}(\rho)}+\rho(\Omega)\right)$ are defined. As in the closed case we now define the analytic torsion of $E_{\rho}$ by

$$
T_{X}(\rho):=\prod_{p=0}^{3} \operatorname{det} \Delta_{p}(\rho)^{(-1)^{p+1 \frac{p}{2}}}
$$

We define a $K$-finite Schwarz-function $k_{t}^{\rho}$ by

$$
k_{t}^{\rho}:=e^{-t \rho(\Omega)} \sum_{p=0}^{3}(-1)^{p} p h_{t}^{\nu_{p}(\rho)} .
$$

Then if we apply equation (4.10), we obtain

$$
\log T_{X}(\rho)=\left.\frac{1}{2} \frac{d}{d z}\left(\frac{1}{\Gamma(z)} \int_{0}^{\infty} t^{z-1}\left(I\left(k_{t}^{\rho}\right)+H\left(k_{t}^{\rho}\right)+T\left(k_{t}^{\rho}\right)+\mathcal{I}\left(k_{t}^{\rho}\right)+J\left(k_{t}^{\rho}\right)\right) d t\right)\right|_{z=0},
$$

where the right hand side is defined near $z=0$ by analytic continuation of the Mellin transform.

\section{The Determinant formula for the Symmetric Selberg zeta function}

In this section we let $\sigma=\sigma_{k}, k \in \mathbb{N}$. We want to relate the symmetric Selberg zeta function $S(s, \sigma)$ to the graded determinant of certain Laplace-type operators.

We consider the differential operator $A(\sigma)$ which was introduced by Bunke and Olbrich for the closed case $[\mathbb{B O}$, section 1.1.3] and which had been used in [ $\mathbb{P A}$, section 7]. Let us briefly recall its definition. We let the $m_{\nu}(\sigma)$ be as in Lemma 2.1. Then one defines a vector bundle $E(\sigma)$ over $X$ and a differential operator $A(\sigma)$ on $E(\sigma)$ by

$$
E(\sigma):=\bigoplus_{\substack{\nu \in \hat{K} \\ m_{\nu}(\sigma) \neq 0}} E_{\nu} ; \quad A(\sigma):=\bigoplus_{\substack{\nu \in \hat{K} \\ m_{\nu}(\sigma) \neq 0}} A_{\nu}+c(\sigma),
$$

where $c(\sigma)$ is as in the preceding section. We define a K-finite Schwarz function $h_{t}^{\sigma}$ by

$$
h_{t}^{\sigma}:=e^{-t c(\sigma)} \sum_{\nu \in \hat{K}} m_{\nu}(\sigma) h_{t}^{\nu},
$$

where the $h_{t}^{\nu}$ are as in the previous section. Then by $\mathbb{P}$, equation 7.4] for $\sigma^{\prime} \in \hat{M}$ one has

$$
\Theta_{\sigma^{\prime}, \lambda}\left(h_{t}^{\sigma}\right)=e^{-t \lambda^{2}}, \text { if } \sigma^{\prime} \in\left\{\sigma, w_{0} \sigma\right\} ; \quad \Theta_{\sigma^{\prime}, \lambda}\left(h_{t}^{\sigma}\right)=0, \text { if } \sigma^{\prime} \notin\left\{\sigma, w_{0} \sigma\right\}
$$

The bundle $E(\sigma)$ admits a grading $E(\sigma)=E^{+}(\sigma) \oplus E^{-}(\sigma)$ defined by the sign of $m_{\nu}(\sigma)$. In this section we study the relative graded determinant of the operators $A(\sigma)+s$. To define this determinant, we start with the following Lemma.

Lemma 5.1. For $\nu \in \hat{K}, m_{\nu}(\sigma) \neq 0$ one has $c(\sigma) \geq b(\nu)$. 
Proof. By Lemma 2.1, and the definition of the $c(\sigma)$, we have $c(\sigma) \geq c\left(\sigma^{\prime}\right)$ for every $\sigma^{\prime} \in \hat{M}$ with $m_{\nu}(\sigma)\left[\nu: \sigma^{\prime}\right] \neq 0, \nu \in \hat{K}$. Moreover, since $\sigma \neq w_{0} \sigma$, the twisted Dirac operator $D(\sigma)$ can be defined as in $[\mathrm{PA}$, section 8, section 9] and it follows from [Pf, Proposition 8.1] that $A(\sigma)=D(\sigma)^{2}$. Thus the eigenvalues of $A(\sigma)$ are nonnegative and the Lemma follows.

Let $s \in \mathbb{C}$ with $\operatorname{Re}(s)>0$. By Proposition 4.1 and Lemma 5.1, for every $\nu \in \hat{K}$ with $m_{\nu}(\sigma) \neq 0$ the relative determinant $\operatorname{det}\left(A_{\nu}+c(\sigma)+s\right) \in \mathbb{C}^{*}$ is defined. Thus we can define the graded determinant $\operatorname{det}_{\mathrm{gr}}(A(\sigma)+s) \in \mathbb{C}^{*}$ of $A(\sigma)+s$ by

$$
\operatorname{det}_{\mathrm{gr}}(A(\sigma)+s):=\prod_{\substack{\nu \in \hat{K} \\ m_{\nu}(\sigma) \neq 0}}\left(\operatorname{det}\left(A_{\nu}+c(\sigma)+s\right)\right)^{m_{\nu}(\sigma)} .
$$

We now study the function $s \mapsto \operatorname{det}_{\mathrm{gr}}\left(A(\sigma)+s^{2}\right), \operatorname{Re}(s)>0, \operatorname{Re}\left(s^{2}\right)>0$. By (4.10) we have

$$
\begin{aligned}
& \log \operatorname{det}_{\mathrm{gr}}\left(A(\sigma)+s^{2}\right) \\
= & -\left.\frac{d}{d z}\right|_{z=0}\left(\frac{1}{\Gamma(z)} \int_{0}^{\infty} t^{z-1} e^{-t s^{2}}\left(I\left(h_{t}^{\sigma}\right)+H\left(h_{t}^{\sigma}\right)+T\left(h_{t}^{\sigma}\right)+\mathcal{I}\left(h_{t}^{\sigma}\right)+J\left(h_{t}^{\sigma}\right)\right) d t\right),
\end{aligned}
$$

where the right hand side is defined near $z=0$ by analytic continuation of the Mellin transform. We will compute the Mellin transform of each summand on the right hand side separately. In the sequel, we shall write $\mathcal{L} \mathcal{M}$ to indicate that the Laplace-Mellin transform of a function is taken, allthoug we take the Laplace-transform in $s^{2}$ rather than in $s$. Firstly, the idenditiy contribution is easily treated.

Proposition 5.2. Let $s \in \mathbb{C}, \operatorname{Re}(s)>0, \operatorname{Re}\left(s^{2}\right)>0$. For $\operatorname{Re}(z)>3 / 2$ the integral

$$
\mathcal{L} \mathcal{M} I(s, z, \sigma):=\int_{0}^{\infty} t^{z-1} e^{-t s^{2}} I\left(h_{t}^{\sigma}\right) d t
$$

converges absolutely. Moreover, $\mathcal{L} \mathcal{M} I(s, z, \sigma)$ has a meromorphic continuation to $z \in \mathbb{C}$ and is regular at $z=0$. Let $\mathcal{L} \mathcal{M} I(s, \sigma):=\left.\mathcal{L} \mathcal{M} I(s, z, \sigma)\right|_{z=0}$. Then one has

$$
\mathcal{L} \mathcal{M} I(s, \sigma)=-4 \pi \operatorname{vol}(X) \int_{0}^{s} P_{\sigma}(r) d r .
$$

Proof. Since the $P_{\sigma}(z)$ are even polynomials in $z$ of degree 2, it follows from (5.17) and a change of variables that $I\left(h_{t}^{\sigma}\right)=a_{0} t^{-\frac{3}{2}}+a_{1} t^{-\frac{1}{2}}$, where $a_{0}, a_{1} \in \mathbb{C}$. Thus for $s \in \mathbb{C}$, $\operatorname{Re}(s)>0, \operatorname{Re}\left(s^{2}\right)>0$ and $\operatorname{Re}(z)>3 / 2$ the function $\mathcal{L} \mathcal{M} I(s, z, \sigma)$ is defined and it extends to a meromorphic function of $z \in \mathbb{C}$ which is regular at $z=0$. Moreover the assignment $s \rightarrow \mathcal{L} \mathcal{M} I(s, 0, \sigma)$ is holomorphic on $\left\{s \in \mathbb{C}: \operatorname{Re}(s)>0, \operatorname{Re}\left(s^{2}\right)>0\right\}$. Applying $\mathbb{F r}$, Lemma 2, Lemma 3], the Lemma is proved for $s \in(0, \infty)$ and thus it also follows for general $s$.

Next we treat the hyperbolic contribution. For our purposes, it suffices to prove the following estimate. 
Proposition 5.3. Let $s \in(\sqrt{2}, \infty)$. Then for every $z \in \mathbb{C}$ the integral

$$
\mathcal{L} \mathcal{M} H(s, z, \sigma):=\int_{0}^{\infty} t^{z-1} e^{-t s^{2}} H\left(h_{t}^{\sigma}\right) d t
$$

converges absolutely and $\mathcal{L} \mathcal{M} H(s, z, \sigma)$ is an entire function of $z$. Let $\mathcal{L} \mathcal{M} H(s, \sigma):=$ $\left.\mathcal{L} \mathcal{M H}(s, z, \sigma)\right|_{z=0}$. Then there exists a constant $C$ such that one has $|\mathcal{L} \mathcal{M} H(s, \sigma)| \leq C s^{-2}$.

Proof. For $\gamma \in \Gamma_{\mathrm{s}}$ we let $\gamma_{0}$ be a generator of $Z(\gamma)$ and we let

$$
f(t):=\sum_{[\gamma] \in \mathrm{C}(\Gamma)_{\mathrm{s}}-[1]} e^{-2 \ell(\gamma)} \ell\left(\gamma_{0}\right) \frac{\operatorname{Tr}(\sigma)\left(m_{\gamma}\right)+\operatorname{Tr}\left(w_{0} \sigma\right)\left(m_{\gamma}\right)}{\operatorname{det}\left(\operatorname{Id}-\left.\operatorname{Ad}\left(m_{\gamma} a_{\gamma}\right)\right|_{\overline{\mathfrak{n}}}\right)} \frac{e^{-\ell(\gamma)^{2} / 4 t}}{(4 \pi t)^{\frac{1}{2}}} .
$$

Then, since $\check{\sigma}=w_{0} \sigma$, by (5.17) and [Pf, equation 5.4] we have $H\left(h_{t}^{\sigma}\right)=f(t)$. Thus by [MP2, Proposition 10.2], it remains to prove the estimate in $s$. By [MP2, equation 10.8] there is $C_{1}$ such that

$$
\int_{1}^{\infty} t^{-1} e^{-t s^{2}}|f(t)| d t \leq C_{1} e^{-\frac{s^{2}}{4}}
$$

Moreover, by [MP2, equation 10.12] there exists a constant $c>0$ such that for $0<t \leq 1$ one can estimate $|f(t)| \leq e^{-\frac{c}{t}}$. Thus, by partial integration we obtain

$$
\int_{0}^{1} t^{-1}|f(t)| e^{-t s^{2}} d t \leq C_{2} \int_{0}^{1} e^{-t s^{2}} e^{-\frac{c}{2 t}} d t=C_{2}\left(-\frac{1}{s^{2}} e^{-s^{2}} e^{-\frac{c}{2}}+\frac{c}{2 s^{2}} \int_{0}^{1} t^{-2} e^{-t s^{2}} e^{-\frac{c}{2 t}} d t\right)
$$

for some constant $C_{2}$. It follows that there exists a constant $C_{3}$ such that

$$
\int_{0}^{1} t^{-1}|f(t)| e^{-t s^{2}} d t \leq C_{3} s^{-2}
$$

This proves the proposition.

The contribution of the distribution $T$ is as follows.

Proposition 5.4. Let $s \in \mathbb{C}, \operatorname{Re}\left(s^{2}\right)>0, \operatorname{Re}(s)>0$. For $\operatorname{Re}(z)>3 / 2$ the integral

$$
\mathcal{L} \mathcal{M T}(s, z, \sigma):=\int_{0}^{\infty} t^{z-1} e^{-t s^{2}} T\left(h_{t}^{\sigma}\right) d t
$$

conveges absolutely. Moreover, the function $z \mapsto \mathcal{L} \mathcal{M T}(s, z, \sigma)$ has a meromorphic continuation to $\mathbb{C}$ which is regular at $0 . \operatorname{Let} \mathcal{L} \mathcal{M} T(s, \sigma):=\left.\mathcal{L} \mathcal{M} T(s, z, \sigma)\right|_{z=0}$. Then one has $\mathcal{L} \mathcal{M T}(s, \sigma)=-2 C(\Gamma) s$.

Proof. By (5.17) and the definition of $T$ one has $\mathcal{L} \mathcal{M} T(s, z, \sigma)=\frac{C(\Gamma)}{\sqrt{\pi}} s^{-2 z+1} \Gamma\left(z-\frac{1}{2}\right)$ and the proposition follows.

For the invariant distribution $\mathcal{I}$ associated to the weighted orbital integral we have the following proposition. 
Proposition 5.5. Let the meromorphic function $\Omega(\sigma, \lambda), \sigma \in \hat{M}, \lambda \in \mathbb{C}$ be defined as in MP2, Theorem 6.2]. Then for $k \in \mathbb{N}$ the function $\Omega\left(\sigma_{k}, \lambda\right)$ is given as

$$
\Omega\left(\sigma_{k}, \lambda\right)=-2 \gamma-\psi(1+i \lambda)-\psi(1-i \lambda)-\sum_{1 \leq l<|k|} \frac{2 l}{\lambda^{2}+l^{2}}-\frac{|k|}{\lambda^{2}+k^{2}},
$$

where $\gamma$ is the Euler-Mascheroni constat and $\psi$ is the Digamma function. Moreover, for $s \in \mathbb{C}, \operatorname{Re}(s)>0, \operatorname{Re}\left(s^{2}\right)>0$ and $z \in \mathbb{C}, \operatorname{Re}(z)>3 / 2$ the integral

$$
\mathcal{L} \mathcal{M I}(s, z, \sigma):=\int_{0}^{\infty} t^{z-1} e^{-t s^{2}} \mathcal{I}\left(h_{t}^{\sigma}\right) d t
$$

converges absolutely. The function $z \mapsto \mathcal{L} \mathcal{M I}(s, z, \sigma)$ has a meromorphic continuation to $z \in \mathbb{C}$ with an at most simple pole at $z=0$. Let $\mathcal{L} \mathcal{M I}(s, \sigma):=\left.\frac{\partial}{\partial z}\right|_{z=0} \frac{\mathcal{L} \mathcal{M}(s, z, \sigma)}{\Gamma(z)}$. Then there exists a constant $C_{0}$ which is independent of $X$ such that for every $k \in \mathbb{N}$ one has

$$
\mathcal{L} \mathcal{M I}\left(s, \sigma_{k}\right)=\kappa(X) C_{0}+2 \kappa(X) \gamma s+2 \kappa(X) \log \Gamma(s+k)+\kappa(X) \log (s+k) .
$$

Proof. The statement about $\Omega\left(\sigma_{k}, \lambda\right)$ follows from an elementary computation using the identity $\psi(z+1)=\frac{1}{z}+\psi(z)$. Thus the Proposition follows from (5.17) and [MP2, Lemma 10.5, Lemma 10.6]. Here we remark that the assumption $c \in(0, \infty)$ in these Lemmas can be weakened to $c \in \mathbb{C}, \operatorname{Re}(c)>0$. The proofs remain the same.

We finally turn to the contribution of the non-invariant distribution $J$.

Proposition 5.6. Let the distribution $J$ be as in (4.9) and let $k \in \mathbb{N}$. The one has

$$
J\left(h_{t}^{\sigma_{k}}\right)=\frac{\kappa(X)}{2 \pi}\left(1+2 \sum_{1 \leq j<|k|} e^{-t\left(k^{2}-j^{2}\right)}+e^{-t k^{2}}\right) \int_{\mathbb{R}} \frac{1}{i \lambda+k} e^{-t \lambda^{2}} d \lambda .
$$

Let $s \in \mathbb{C}, \operatorname{Re}(s)>0, \operatorname{Re}\left(s^{2}\right)>0$. For $\operatorname{Re}(z)>3 / 2$ the integral

$$
\mathcal{L} \mathcal{M} J\left(s, z, \sigma_{k}\right):=\int_{0}^{\infty} t^{z-1} e^{-t s^{2}} J\left(h_{t}^{\sigma_{k}}\right) d t
$$

converges absolutely. Moreover, the function $z \mapsto \mathcal{L} \mathcal{M} J\left(s, z, \sigma_{k}\right)$ has a meromorphic continuation to $\mathbb{C}$ with a simple pole at 0 . For $\mathcal{L} \mathcal{M} J\left(s, \sigma_{k}\right):=\left.\frac{\partial}{\partial z}\right|_{z=0} \frac{\mathcal{L} \mathcal{M} J\left(s, z, \sigma_{k}\right)}{\Gamma(z)}$ one has

$$
\begin{aligned}
\mathcal{L} \mathcal{M} J\left(s, \sigma_{k}\right)= & -2 \kappa(X) \sum_{1 \leq j<k} \log \left(\sqrt{s^{2}+k^{2}-j^{2}}+k\right) \\
& -\kappa(X) \log \left(\sqrt{s^{2}+k^{2}}+k\right)-\kappa(X) \log (s+k) .
\end{aligned}
$$

Proof. For $j \in \mathbb{Z}, l \in \mathbb{N}^{0},|j| \leq l$ we let $c_{\nu_{l}}\left(\sigma_{j}, z\right)$ be the Harish-Chandra $c$-function associated to the representation $\nu_{l}$ and $\sigma_{j}$ defined by [MP2, equation 6.7]. Then by [Co, Appendix 2] one has

$$
c_{\nu_{l}}\left(\sigma_{j}, z\right):=\frac{\Gamma(i z-j) \Gamma(i z+j)}{\Gamma(i z-l) \Gamma(i z+l+1)}
$$


see also [MP2, equation 6.8]. By [MP2, equation 6.14] and the definition of $h_{t}^{\sigma_{k}}$ one has

$$
J\left(h_{t}^{\sigma_{k}}\right)=-\frac{\kappa(X) e^{-t c\left(\sigma_{k}\right)}}{4 \pi i} \sum_{\nu \in \hat{K}} \sum_{\sigma^{\prime} \in \hat{M}} m_{\nu}\left(\sigma_{k}\right)\left[\nu: \sigma^{\prime}\right] \int_{D_{\epsilon}} e^{-t\left(\zeta^{2}-c\left(\sigma^{\prime}\right)\right)} c_{\nu}\left(\sigma^{\prime}: \zeta\right)^{-1} \frac{d}{d \zeta} c_{\nu}\left(\sigma^{\prime}: \zeta\right) d \zeta
$$

Thus if one applies Lemma 2.1, equation (5.19) follows. If one applies [MP2, Lemma 10.5] to (5.19), the formula for $\mathcal{L} \mathcal{M} J\left(s, \sigma_{k}\right)$ follows. Here we remark again that the condition $c \in(0, \infty)$ in [MP2, Lemma 10.5] can be weakened to the condition $c \in \mathbb{C}, \operatorname{Re}(c)>0$ without changing the proof.

By the preceding proposition, each summand on the right hand side of (5.18) can be integrated individually and we have

$$
\begin{aligned}
& \log \operatorname{det}_{\mathrm{gr}}\left(A(\sigma)+s^{2}\right) \\
= & -\mathcal{L} \mathcal{M} I(s, \sigma)-\mathcal{L} \mathcal{M} H(s, \sigma)-\mathcal{L} \mathcal{M} T(s, \sigma)-\mathcal{L} \mathcal{M I}(s, \sigma)-\mathcal{L} \mathcal{M} J(s, \sigma)
\end{aligned}
$$

To proof our determinant formula, we will also need the following Lemma.

Lemma 5.7. Let $\operatorname{Tr}_{\mathrm{reg}}\left(e^{-t A(\sigma)}\right):=\sum_{\nu \in \hat{K}} m_{\nu}(\sigma) e^{-t c(\sigma)} \operatorname{Tr}_{\mathrm{reg}}\left(e^{-t A_{\nu}}\right)$. Then for $s, s_{1} \in \mathbb{C}$ with $\operatorname{Re}(s)>0, \operatorname{Re}\left(s_{1}\right)>0$ one has

$$
\begin{aligned}
& \int_{0}^{\infty}\left(e^{-t s}-e^{-t s_{1}}\right) \operatorname{Tr}_{\mathrm{reg}}\left(e^{-t A(\sigma)}\right) d t \\
= & \left.\frac{d}{d \zeta} \log \operatorname{det}_{\mathrm{gr}}(A(\sigma)+\zeta)\right|_{\zeta=s}-\left.\frac{d}{d \zeta} \log \operatorname{det}_{\mathrm{gr}}(A(\sigma)+\zeta)\right|_{\zeta=s_{1}}
\end{aligned}
$$

Proof. For $\zeta \in \mathbb{C}, \operatorname{Re}(\zeta)>0$ and $z \in \mathbb{C}$ let $\xi_{A(\sigma)}(\zeta, z):=\sum_{\nu \in \hat{K}} m_{\nu}(\sigma) \xi_{\nu}(\zeta+c(\sigma), z)$, where the $\xi_{\nu}$ are as in the previous section. Then by definition one has

$$
\log \left(\operatorname{det}_{\mathrm{gr}}(A(\sigma)+s)\right)=-\left.\frac{\partial}{\partial z} \frac{\xi_{A(\sigma)}(s, z)}{\Gamma(z)}\right|_{z=0}
$$

By (4.7) and the choice of $s$ and $s_{1},\left(e^{-t s}-e^{-t s_{1}}\right) \operatorname{Tr}_{\text {reg }}\left(e^{-t A(\sigma)}\right)$ decays exponentially for $t \rightarrow \infty$ and by (4.12) one has $\left(e^{-t s}-e^{-t s_{1}}\right) \operatorname{Tr}_{\mathrm{reg}}\left(e^{-t A(\sigma)}\right)=O\left(t^{-1 / 2}\right)$ as $t \rightarrow 0$. Thus the integral in the lemma exists. By Proposition 4.1, there exists a constant $\alpha(\sigma)$ such that for all $\zeta$ with $\operatorname{Re}(\zeta)>0$ one has $\left.\operatorname{Res}\right|_{z=0} \xi_{A(\sigma)}(\zeta, z)=\alpha(\sigma)$ and since $\frac{1}{\Gamma(z)}=z+\gamma z^{2}+O\left(z^{3}\right)$ as $z \rightarrow 0$, for all such $\zeta$ one has $\log \operatorname{det}_{\mathrm{gr}}(A(\sigma)+\zeta)=-\lim _{z \rightarrow 0}\left(\xi_{A(\sigma)}(\zeta, z)-\frac{\alpha(\sigma)}{z}+\gamma \alpha(\sigma)\right)$. One has $\xi_{A(\sigma)}(\zeta, z+1)=-\frac{\partial}{\partial \zeta} \xi_{A(\sigma)}(\zeta, z)$ by the definition of $\xi_{A(\sigma)}$ and by meromorphic 
continuation. Thus one has

$$
\begin{aligned}
& \int_{0}^{\infty}\left(e^{-t s}-e^{-t s_{1}}\right) \operatorname{Tr}_{\mathrm{reg}}\left(e^{-t A(\sigma)}\right) d t=\lim _{z \rightarrow 0} \int_{0}^{\infty} t^{z}\left(e^{-t s}-e^{-t s_{1}}\right) \operatorname{Tr}_{\mathrm{reg}}\left(e^{-t A(\sigma)}\right) d t \\
= & -\lim _{z \rightarrow 0}\left(\left.\frac{\partial}{\partial \zeta} \xi_{A(\sigma)}(\zeta, z)\right|_{\zeta=s}-\left.\frac{\partial}{\partial \zeta} \xi_{A(\sigma)}(\zeta, z)\right|_{\zeta=s_{1}}\right) \\
= & -\lim _{z \rightarrow 0}\left(\left.\frac{\partial}{\partial \zeta}\left(\xi_{A(\sigma)}(\zeta, z)-\frac{\alpha(\sigma)}{z}+\gamma \alpha(\sigma)\right)\right|_{\zeta=s}-\left.\frac{\partial}{\partial \zeta}\left(\xi_{A(\sigma)}(\zeta, z)-\frac{\alpha(\sigma)}{z}+\gamma \alpha(\sigma)\right)\right|_{\zeta=s_{1}}\right) \\
= & \left.\frac{d}{d \zeta} \log \operatorname{det}_{\mathrm{gr}}(A(\sigma)+\zeta)\right|_{\zeta=s}-\left.\frac{d}{d \zeta} \log \operatorname{det}_{\mathrm{gr}}(A(\sigma)+\zeta)\right|_{\zeta=s_{1}} .
\end{aligned}
$$

Here limit and differentation in the third line can be interchanged since the function $(\zeta, z) \mapsto \xi_{A(\sigma)}(\zeta, z)-\frac{\alpha(\sigma)}{z}+\gamma \alpha(\sigma)$ is holomorphic for $\zeta \in \mathbb{C}, \operatorname{Re}(\zeta)>0$ and $z$ in a neighbourhood of zero.

Now we can state the determinant formula for the symmetric Selberg zeta function, which is the main result of this section.

Proposition 5.8. Let $s \in \mathbb{C}, \operatorname{Re}(s)>0, \operatorname{Re}\left(s^{2}\right)>0$. Let $\kappa(X)$ be the number of cusps of $X$ and let $c_{\Gamma}:=2(C(\Gamma)-\gamma \kappa(X))$, where $C(\Gamma)$ is as above. Then there exists a constant $C_{0}$ which is independent of $X$ such that for every $k \in \mathbb{N}$ one has

$$
\begin{aligned}
S\left(s, \sigma_{k}\right)= & e^{\kappa(X) C_{0}} \operatorname{det}_{\mathrm{gr}}\left(A(\sigma)+s^{2}\right) \exp \left(-4 \pi \operatorname{vol}(X) \int_{0}^{s} P_{\sigma_{k}}(r) d r\right) \Gamma^{2 \kappa(X)}(s+k) \\
& \cdot(s+k)^{\kappa(X)} \exp \left(\mathcal{L} \mathcal{M} J\left(s, \sigma_{k}\right)-s c_{\Gamma}\right) .
\end{aligned}
$$

Proof. We fix $s_{1} \in \mathbb{R}, s_{1}>2$ and let $s \in \mathbb{R}, s>2$. We let $\sigma:=\sigma_{k}$. Then we can apply $[\mathbb{P A}$, equation 7.7] with $N=2, s_{2}:=s$ and obtain

$$
\int_{0}^{\infty}\left(e^{-t s^{2}}-e^{-t s_{1}^{2}}\right) H\left(h_{t}^{\sigma}\right) d t=\frac{1}{2 s} \frac{d}{d s} S(s, \sigma)-\frac{1}{2 s_{1}} \frac{d}{d s} S\left(s_{1}, \sigma\right) .
$$

Thus if we apply (4.10), (5.17) and equation (4.8), Proposition 5.2, Proposition 5.4, Proposition 5.5 and Propostion 5.6, we obtain a constant $a$, depending on $s_{1}$, such that

$$
\begin{aligned}
& \int_{0}^{\infty}\left(e^{-t s^{2}}-e^{-t s_{1}^{2}}\right) \operatorname{Tr}_{\mathrm{reg}}\left(e^{-t A(\sigma)}\right) d t \\
= & \frac{1}{2 s} \frac{d}{d s} \log S(s, \sigma)+\frac{2 \pi \operatorname{vol}(X) P_{\sigma}(s)}{s}+\frac{C(\Gamma)}{s}-\frac{\kappa(X) \gamma+\kappa(X) \psi(s+k)}{s} \\
& +\frac{\kappa(X)}{2 \sqrt{s^{2}+k^{2}}\left(\sqrt{s^{2}+k^{2}}+k\right)}+\sum_{1 \leq j<k} \frac{\kappa(X)}{\sqrt{s^{2}+k^{2}-j^{2}}\left(\sqrt{s^{2}+k^{2}-j^{2}}+k\right)}+a \\
= & \frac{1}{2 s} \frac{d}{d s}(\log S(s, \sigma)-\mathcal{L} \mathcal{M} I(s, \sigma)-\mathcal{L} \mathcal{M T}(s, \sigma)-\mathcal{L} \mathcal{M I}(s, \sigma)-\mathcal{L} \mathcal{M} J(s, \sigma))+a .
\end{aligned}
$$


If we multiply this equation by $2 s$ and apply Lemma 5.7, we obtain

$$
\begin{aligned}
\log S(s, \sigma)= & \log \operatorname{det}_{\mathrm{gr}}\left(A(\sigma)+s^{2}\right)+\mathcal{L} \mathcal{M} I(s, \sigma)+\mathcal{L} \mathcal{M I}(s, \sigma)+\mathcal{L} \mathcal{M} T(s, \sigma)+\mathcal{L} \mathcal{M} J(s, \sigma) \\
& +a s^{2}+b .
\end{aligned}
$$

for some constant $b$. Thus by (5.20) we have $\log S(s, \sigma)=-\mathcal{L} \mathcal{M} H(s, \sigma)+a s^{2}+b$. Applying [Pf, equations 3.3, 3.4, 3.5], it follows that $\log S(s, \sigma)$ decays exponentially as $\operatorname{Re}(s) \rightarrow \infty$. Since $\mathcal{L} \mathcal{M} H(s, \sigma)$ tends to zero for $s \in \mathbb{R}, s \rightarrow \infty$ by Propostion 5.3, the constants $a$ and $b$ are zero. If we apply Proposition 5.2, Proposition 5.4 and Proposition 5.5 and to the right hand side of (5.21), the Proposition follows for $s \in(2, \infty)$. By $[\mathbb{P t}$, Theorem 1.1], the function $S(s, \sigma)$ has a meromorphic continuation to $\mathbb{C}$ and since all functions on the right hand side of the equation in the Proposition are holomorphic in $s \in \mathbb{C}, \operatorname{Re}(s)>0$, $\operatorname{Re}\left(s^{2}\right)>0$ by Proposition 4.1, Lemma 5.1 and Proposition 5.6, the Proposition follows.

\section{The FUnCTIONAL EQUATIONS}

Let $\sigma \in \hat{M}$. In this section we prove a functional equation for the symmetric Selberg zeta function $S(s, \sigma)$.

For $\nu \in \hat{K}$ and $\sigma \in \hat{M}$ with $[\nu: \sigma] \neq 0$ we define the space $\mathcal{E}(\nu: \sigma)$ and the operator $\mathbf{C}(\nu: \sigma: \lambda): \mathcal{E}(\nu: \sigma) \rightarrow \mathcal{E}\left(\nu: w_{0} \sigma\right)$ as in [Pf, section 4]. Let us first symmetrize the scattering matrices. For $\sigma \in \hat{M}, \sigma \neq w_{0} \sigma$ and $\nu \in \hat{K}$ we let $\overline{\mathcal{E}}(\boldsymbol{\sigma}, \nu):=\mathcal{E}(\boldsymbol{\sigma}, \nu) \oplus \mathcal{E}\left(\boldsymbol{w}_{0} \boldsymbol{\sigma}, \nu\right)$ and for $s \in \mathbb{C}$ we let

$$
\overline{\mathbf{C}}(\sigma: \nu: s): \overline{\mathcal{E}}(\boldsymbol{\sigma}, \nu) \rightarrow \overline{\mathcal{E}}(\boldsymbol{\sigma}, \nu) ; \quad \overline{\mathbf{C}}(\sigma: \nu: s):=\left(\begin{array}{cc}
0 & \mathbf{C}\left(w_{0} \sigma: \nu: s\right) \\
\mathbf{C}(\sigma: \nu: s) & 0
\end{array}\right) .
$$

By the arguments of $\mathbb{P A}$, section 4], the function $(\operatorname{det} \overline{\mathbf{C}}(\sigma: \nu: s))^{\frac{1}{\operatorname{dim}(\nu)}}$ is canonically defined. For $\sigma \in \hat{M}$ we let $\nu_{\sigma}$ be as in $\left[\mathbb{P f}\right.$, section 4]. Then, if $\sigma=\sigma_{k}$ we have $\nu_{\sigma}=\nu_{|k|}$. To save notation, for $k \in \frac{1}{2} \mathbb{N}$ we shall write

$$
\left(\operatorname{det} \overline{\mathbf{C}}\left(\sigma_{k}: \nu_{k}: s\right)\right)^{\frac{1}{\operatorname{dim}(\nu)}}=: \mathbf{C}(k: s) .
$$

Then $\mathbf{C}(k: s)$ is a meromorphic function of $s$ which has no zeroes and poles for $s \in i \mathbb{R}$. By [ $[\overline{P f}$, equation 4.2] it satisfies $\mathbf{C}(k: s) \mathbf{C}(k:-s)=1$.

We can now state a functional equation for the symmetric Selberg zeta function.

Proposition 6.1. Let $k \in \mathbb{N}$ and let $c_{\Gamma}$ be as in Proposition 5.8. Then the symmetric Selberg zeta function $S\left(s, \sigma_{k}\right)$ satisfies the functional equation

$$
S\left(-s, \sigma_{k}\right)=S\left(s, \sigma_{k}\right) \exp \left(8 \pi \operatorname{vol}(X) \int_{0}^{s} P_{\sigma_{k}}(r) d r+2 c_{\Gamma} s\right) \frac{(\Gamma(-s+k))^{2 \kappa(X)}}{(\Gamma(s+k))^{2 \kappa(X)}} \frac{\mathbf{C}(k: s)}{\mathbf{C}(k: 0)} .
$$

Proof. Let

$$
\Xi\left(s, \sigma_{k}\right):=\exp \left(4 \pi \operatorname{vol}(X) \int_{0}^{s} P_{\sigma_{k}}(r) d r+s c_{\Gamma}\right)(\Gamma(s+k))^{-2 \kappa(X)} \cdot S\left(s, \sigma_{k}\right) .
$$


We note that the Polynomial $Q\left(\sigma_{k}, \lambda\right)$ and the constants $c_{j, l}(\sigma)$ occuring in $[\mathbb{P A}$, Proposition 5.4] are zero in the 3 -dimensional case. Thus if we combine $[\mathbb{P A}$, Proposition 7.2], $[\mathrm{PA}$, equation 4.2] [Pf, equation 4.10] and [PA], Remark 4.3], we obtain

$$
\frac{\Xi^{\prime}\left(s, \sigma_{k}\right)}{\Xi\left(s, \sigma_{k}\right)}+\frac{\Xi^{\prime}\left(-s, \sigma_{k}\right)}{\Xi\left(-s, \sigma_{k}\right)}=-\frac{d}{d s} \log \mathbf{C}(k: s) .
$$

Hence the logarithmic derivative of $\frac{\Xi(s, \sigma)}{\Xi(-s, \sigma)} \mathbf{C}(k: s)$ is zero and so this function is constant. Now the order of the singularity of the function $\Xi\left(s, \sigma_{k}\right)$ at 0 is the same as the order of the singularity of $S(s, \sigma)$ at 0 . This order is even by [ [Pf, Theorem 9.2]. Since $P_{\sigma_{k}}(r)$ is an even polynomial, the proposition follows.

The previous proposition implies the following functional equation for the symmetric Ruelle zeta function.

Proposition 6.2. Let $k \in \mathbb{N}$. Then the symmetric Ruelle zeta function $R_{\mathrm{sym}}\left(s, \sigma_{k}\right)$ satisfies the functional equation

$$
\begin{aligned}
R_{\mathrm{sym}}\left(-s, \sigma_{k}\right)= & R_{\mathrm{sym}}\left(s, \sigma_{k}\right) \exp \left(-\frac{8}{\pi} \operatorname{vol}(X) s\right) \frac{\mathbf{C}(k: s-1) \mathbf{C}(k: s+1)}{\mathbf{C}(k+1: s) \mathbf{C}(k-1: s)} \\
& \cdot \frac{\mathbf{C}(k+1: 0) \mathbf{C}(k-1: 0)}{\mathbf{C}(k: 0)^{2}}
\end{aligned}
$$

Proof. The same argument as in [Mü3, Lemma 3.1] gives

$$
R_{\mathrm{sym}}\left(s, \sigma_{k}\right)=\frac{S\left(s+1, \sigma_{k}\right) S\left(s-1, \sigma_{k}\right)}{S\left(s, \sigma_{k+1}\right) S\left(s, \sigma_{k-1}\right)} .
$$

Moreover, using (4.8) we compute

$$
\int_{0}^{s+1} P_{\sigma_{k}}(r) d r+\int_{0}^{s-1} P_{\sigma_{k}}(r) d r-\int_{0}^{s} P_{\sigma_{k+1}}(r) d r-\int_{0}^{s} P_{\sigma_{k-1}}(r) d r=-\frac{s}{\pi^{2}} .
$$

Thus the proposition follows from Proposition 6.1.

To prove Theorem 1.2, we will also need the following proposition.

Proposition 6.3. Let $m \in \mathbb{N}, m \geq 3$. Then

$$
\begin{aligned}
R_{\rho(m)}(s) R_{\rho(m)_{\theta}}(s)= & R_{\rho(2)}(s) R_{\rho(2){ }_{\theta}}(s) \frac{\mathbf{C}(m: m+1-s)}{\mathbf{C}(m+1: m-s)} \frac{\mathbf{C}(3: 2-s)}{\mathbf{C}(2: 3-s)} \frac{\mathbf{C}(m+1: 0)}{\mathbf{C}(m: 0)} \frac{\mathbf{C}(2: 0)}{\mathbf{C}(3: 0)} \\
& \cdot \prod_{k=3}^{m} R_{\mathrm{sym}}\left(k-s, \sigma_{k}\right) R_{\mathrm{sym}}\left(k+s, \sigma_{k}\right) \exp \left(-\frac{8}{\pi} \operatorname{vol}(X)(k-s)\right) .
\end{aligned}
$$

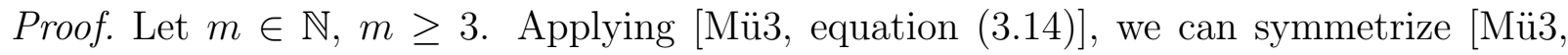
equation (8.2)] and obtain

$$
R_{\rho(m)}(s) R_{\rho(m)_{\theta}}(s)=R_{\rho(2)}(s) R_{\rho(2)_{\theta}}(s) \prod_{k=3}^{m} R_{\mathrm{sym}}\left(s+k, \sigma_{k}\right) R_{\mathrm{sym}}\left(s-k, \sigma_{k}\right) .
$$

Thus together with proposition 6.2, the proposition follows. 


\section{Proof of the MAin Results}

In this section we prove our main results. Let $m \in \mathbb{N}$. Arguing as in [Mü3, Proposition 3.5] it follows that

$$
R_{\rho(m)}(s) R_{\rho(m)_{\theta}}(s)=\frac{S\left(s+m+1, \sigma_{m}\right) S\left(s-m-1, \sigma_{m}\right)}{S\left(s+m, \sigma_{m+1}\right) S\left(s-m, \sigma_{m+1}\right)} .
$$

Now we express the analytic torsion $T_{X}(\rho(m))$ by the graded determinants associated to the operators $A(\sigma)$ which were introduced section 5. Namely, we have the following Proposition which is a generalization of Mü3, equation 7.28] to the noncompact case.

Proposition 7.1. Let $m \in \mathbb{N}$. Then one has

$$
T_{X}(\rho(m))^{2}=\frac{\operatorname{det}_{\mathrm{gr}}\left(A\left(\sigma_{m}\right)+(m+1)^{2}\right)}{\operatorname{det}_{\mathrm{gr}}\left(A\left(\sigma_{m+1}\right)+m^{2}\right)} .
$$

Proof. Let $k_{t}^{\rho(m)}$ be as in (4.13). Then by Lemma 2.2 and Remark 2.3, as a special case of [MP2, Proposition 8.2] one has $k_{t}^{\rho(m)}=e^{-t(m+1)^{2}} h_{t}^{\sigma_{m}}-e^{-t m^{2}} h_{t}^{\sigma_{m+1}}$. Applying (4.14) and (5.18), the proposition follows.

In order to relate the behaviour of $R_{\rho}(m) R_{\rho(m)_{\theta}}$ at 0 to the analytic torsion $T_{X}(\rho(m))$, we want to apply the determinant formula for the symmetric Selberg zeta function from Proposition 5.8 to the right hand side of (7.22) and combine it with Proposition 7.1.

However, in contrast to a closed hyperbolic manifold, this is not possible directly since the determinant formula for the symmetric Selberg zeta function is valid only for $s \in \mathbb{C}$ with $\operatorname{Re}(s)>0, \operatorname{Re}\left(s^{2}\right)>0$. Thus we first have to apply the functional equation from Proposition 6.1. We obtain the following proposition.

Proposition 7.2. For $m \in \mathbb{N}$ one has

$$
\begin{aligned}
R_{\rho(m)}(s) R_{\rho(m)_{\theta}}(s)= & e^{2 c_{\Gamma}} \frac{S\left(-s+m+1, \sigma_{m}\right) S\left(s+m+1, \sigma_{m}\right) \mathbf{C}(m+1: 0)}{S\left(-s+m, \sigma_{m+1}\right) S\left(s+m, \sigma_{m+1}\right) \mathbf{C}(m: 0)} \\
& \cdot \frac{\mathbf{C}(m: m+1-s)(\Gamma(s-1))^{2 \kappa(X)} \exp \left(8 \pi \operatorname{vol}(X) \int_{0}^{-s+m+1} P_{\sigma_{m}}(r) d r\right)}{\mathbf{C}(m+1: m-s)(\Gamma(s+1))^{2 \kappa(X)} \exp \left(8 \pi \operatorname{vol}(X) \int_{0}^{-s+m} P_{\sigma_{m+1}}(r) d r\right)} .
\end{aligned}
$$

Proof. By proposition 6.1 we have

$$
\begin{aligned}
\frac{S\left(s-m-1, \sigma_{m}\right)}{S\left(s-m, \sigma_{m+1}\right)}= & e^{2 c_{\Gamma}} \frac{S\left(-s+m+1, \sigma_{m}\right) \exp \left(8 \pi \operatorname{vol}(X) \int_{0}^{-s+m+1} P_{\sigma_{m}}(r) d r\right)}{S\left(-s+m, \sigma_{m+1}\right) \exp \left(8 \pi \operatorname{vol}(X) \int_{0}^{-s+m} P_{\sigma_{m+1}}(r) d r\right)} \\
& \frac{\mathbf{C}(m+1: 0) \mathbf{C}(m: m+1-s)(\Gamma(s-1))^{2 \kappa(X)}}{\mathbf{C}(m: 0) \mathbf{C}(m+1: m-s)(\Gamma(s+1))^{2 \kappa(X)}} .
\end{aligned}
$$

Applying (7.22), the proposition follows. 
Now we can prove Proposition 1.3. We shall state the proposition also for $m+\frac{1}{2}, m \in \mathbb{N}$. The proof remains the same if one makes the appropriate modifications in section 5 and in Proposition 6.3, Proposition 7.1 and Proposition 7.2. For $m \in \mathbb{N}$ we define

$$
c(m+1 / 2):=\frac{\prod_{j=0}^{m-1} \sqrt{(m+3 / 2)^{2}+(m+1 / 2)^{2}-(j+1 / 2)^{2}}+m+1 / 2}{\prod_{j=0}^{m} \sqrt{(m+3 / 2)^{2}+(m+1 / 2)^{2}-(j+1 / 2)^{2}}+m+3 / 2} .
$$

Then we have the following proposition.

Proposition 7.3. For $m \in \mathbb{N}$ we define the constant $c(m) s$ and $c\left(m+\frac{1}{2}\right)$ as in Theorem 1.1 resp. equation (7.23). Then one has

$$
\begin{aligned}
& T_{X}(\rho(m))^{4} \\
= & c(m)^{4 \kappa(X)} \frac{\mathbf{C}(m: 0)}{\mathbf{C}(m+1: 0)} \lim _{s \rightarrow 0}\left(R_{\rho(m)}(s) R_{\rho(m)_{\theta}}(s) \frac{\mathbf{C}(m+1: m-s)}{\mathbf{C}(m: m+1-s)}(\Gamma(s-1))^{-2 \kappa(X)}\right)
\end{aligned}
$$

and

$$
\begin{aligned}
T_{X}(\rho(m+1 / 2))^{4}= & c(m+1 / 2)^{4 \kappa(X)} \frac{\mathbf{C}(m+1 / 2: 0)}{\mathbf{C}(m+3 / 2: 0)} \cdot \lim _{s \rightarrow 0}\left(R_{\rho(m+1 / 2)}(s) R_{\rho(m+1 / 2)_{\theta}}(s)\right. \\
& \left.\frac{\mathbf{C}(m+3 / 2: m+1 / 2-s)}{\mathbf{C}(m+1 / 2: m+3 / 2-s)}(\Gamma(s-1))^{-2 \kappa(X)}\right) .
\end{aligned}
$$

Proof. Let $m \in \mathbb{N}$. To save notation, let us first introduce two auxiliary functions. Let

$$
\begin{aligned}
P_{\rho(m)}(s):= & \exp \left(-4 \pi \operatorname{vol}(X) \int_{0}^{s+m+1} P_{\sigma_{m}}(r) d r+4 \pi \operatorname{vol}(X) \int_{0}^{-s+m+1} P_{\sigma_{m}}(r) d r\right. \\
& \left.-4 \pi \operatorname{vol}(X) \int_{0}^{-s+m} P_{\sigma_{m+1}}(r) d r+4 \pi \operatorname{vol}(X) \int_{0}^{s+m} P_{\sigma_{m+1}}(r) d r\right) .
\end{aligned}
$$

Moreover, let

$$
\begin{aligned}
J_{\rho(m)}(s):=\exp ( & \mathcal{L} \mathcal{M} J\left(-s+m, \sigma_{m+1}\right)+\mathcal{L} \mathcal{M} J\left(s+m, \sigma_{m+1}\right) \\
& \left.\quad-\mathcal{L} \mathcal{M} J\left(-s+m+1, \sigma_{m}\right)-\mathcal{L} \mathcal{M} J\left(s+m+1, \sigma_{m}\right)\right) .
\end{aligned}
$$


Then by Proposition 7.2, Proposition 5.8 and Proposition 7.1 one has

$$
\begin{aligned}
& \frac{\mathbf{C}(m: 0)}{\mathbf{C}(m+1: 0)} \lim _{s \rightarrow 0}\left(R_{\rho(m)}(s) R_{\rho(m)}(s) \frac{\mathbf{C}(m+1: m-s)(\Gamma(s+1))^{2 \kappa(X)}}{\mathbf{C}(m: m+1-s)(\Gamma(s-1))^{2 \kappa(X)}} J_{\rho(m)}(s)\right) \\
= & \lim _{s \rightarrow 0}\left(e^{2 c_{\Gamma}} \frac{S\left(s+m+1, \sigma_{m}\right) S\left(-s+m+1, \sigma_{m}\right)}{S\left(s+m, \sigma_{m+1}\right) S\left(-s+m, \sigma_{m+1}\right)} J_{\rho(m)}(s)\right) \\
& \cdot \lim _{s \rightarrow 0} \exp \left(8 \pi \operatorname{vol}(X) \int_{0}^{-s+m+1} P_{\sigma_{m}}(r) d r-8 \pi \operatorname{vol}(X) \int_{0}^{-s+m} P_{\sigma_{m+1}}(r) d r\right) \\
= & \lim _{s \rightarrow 0} \frac{\operatorname{det}_{\mathrm{gr}}\left(A\left(\sigma_{m}\right)+(s+m+1)^{2}\right) \operatorname{det}_{\mathrm{gr}}\left(A\left(\sigma_{m}\right)+(-s+m+1)^{2}\right)}{\operatorname{det}_{\mathrm{gr}}\left(A\left(\sigma_{m+1}\right)+(s+m)^{2}\right) \operatorname{det}_{\mathrm{gr}}\left(A\left(\sigma_{m+1}\right)+(-s+m)^{2}\right)} P_{\rho(m)}(s) \\
= & \frac{\operatorname{det}_{\mathrm{gr}}^{2}\left(A\left(\sigma_{m}\right)+(m+1)^{2}\right)}{\operatorname{det}_{\mathrm{gr}}^{2}\left(A\left(\sigma_{m+1}\right)+m^{2}\right)} \\
= & T_{X}(\rho(m))^{4} .
\end{aligned}
$$

Here we used that the function $P_{\rho(m)}(s)$ is an entire function of $s$ satisfying $P_{\rho(m)}(0)=1$. Now by Proposition 5.6 the function $J_{\rho(m)}(s)$ is entire for $s$ in a neighbourhood of zero and one has $J_{\rho(m)}(0)=c(m)^{4 \kappa(X)}$. This proves the proposition for $m \in \mathbb{N}$. For $m+1 / 2$ one can argue in the same way.

Let us finally turn to the proof of Theorem 1.2. We recall that the infinite products in (3.4) defining the Ruelle zeta functions $R(s, \sigma)$ converge absolutely for $\operatorname{Re}(s)>2$. Let $m \in \mathbb{N}, m \geq 3$. By Proposition 7.3 and Proposition 6.3 we have

$$
\begin{aligned}
& T_{X}(\rho(m))^{4} \\
= & c(m)^{4 \kappa(X)} \frac{\mathbf{C}(m: 0)}{\mathbf{C}(m+1: 0)} \lim _{s \rightarrow 0}\left(R_{\rho(m)}(s) R_{\rho(m)_{\theta}}(s) \frac{\mathbf{C}(m+1: m-s)}{\mathbf{C}(m: m+1-s)}(\Gamma(s-1))^{-2 \kappa(X)}\right) \\
= & c(m)^{4 \kappa(X)} \frac{\mathbf{C}(2: 0)}{\mathbf{C}(3: 0)} \lim _{s \rightarrow 0}\left(R_{\rho(2)}(s) R_{\rho(2)_{\theta}}(s) \frac{\mathbf{C}(3: 2-s)}{\mathbf{C}(2: 3-s)}(\Gamma(s-1))^{-2 \kappa(X)}\right) \\
& \cdot \prod_{k=3}^{m} \exp \left(-\frac{8}{\pi} \operatorname{vol}(X) k\right) R_{\mathrm{sym}}\left(k, \sigma_{k}\right)^{2} \\
= & \frac{c(m)^{4 \kappa(X)}}{c(2)^{4 \kappa(X)}} T_{X}(\rho(2))^{4} \exp \left(-\frac{4}{\pi} \operatorname{vol}(X)(m(m+1)-6)\right) \prod_{k=3}^{m} R_{\mathrm{sym}}\left(k, \sigma_{k}\right)^{2} .
\end{aligned}
$$

Now one has $\bar{\sigma}=w_{0} \sigma$ and so by the definition of the Ruelle zeta function and by meromorphic continuation one gets $\overline{R\left(\bar{s}, w_{0} \sigma\right)}=R(s, \sigma)$. Thus one has $R_{\mathrm{sym}}\left(k, \sigma_{k}\right)=\left|R\left(k, \sigma_{k}\right)\right|^{2}$. This proves the first equation in Theorem 1.2. Modifying Proposition 6.3, the second equation in this theorem is obtained in the same way.

\section{REFERENCES}

[BO] U.Bunke, M. Olbrich, Selberg zeta and theta functions, Akademie Verlag, Berlin (1995) 
[Br] U. Bröcker, Die Ruellesche Zetafunktion für G-induzierte Anosov-Fluesse, Ph.D. thesis, Humboldt-Universität Berlin, Berlin, 1998.

[BV] N. Bergeron, A. Venkatesh, The asymptotic growth of torsion homology for arithmetic groups, http://arxiv.org/abs/1004.1083 (2010).

[Che] J. Cheeger, Analytic torsion and the heat equation, Ann. of Math. (2) 109 (1979), 259-322

[Co] L. Cohn,Analytic theory of the Harish-Chandra C-function, Lecture Notes in Mathematics, Vol. 429. Springer-Verlag, Berlin-New York, 1974.

[Do] H. Donnelly, On the point spectrum for finite volume symmetric spaces of negative curvature, Communications in Partial Differential Equations 6 (1981), no. 9, 963-992

[Fr] D. Fried, Analytic torsion and closed geodesics on hyperbolic manifolds, Invent. Math. 84 (1986), no. $3,523-540$

[Gi] P. Gilkey, Invariance theory, the heat equation, and the Atiyah-Singer index theorem, Second edition, Studies in Advanced Mathematics, CRC Press, Boca Raton, FL, 1995.

[GP] Y. Gon, J. Park, The zeta functions of Ruelle and Selberg for hyperbolic manifolds with cusps, Math. Ann. 346, no. 3, (2010), 719-767

[Ho1] W. Hoffmann, The Fourier transform of weighted orbital integrals on semisimple groups of real rank one, J. Reine Angew. Math. 489 (1997), 53-97

[Ho2] W. Hoffmann, An invariant trace formula for rank one lattices, Math. Nachr. 207 (1999), 93-131

[MtM] Matsushima, Murakami, On vector bundle valued harmonic forms and automorphic forms on symmetric riemannian manifolds, Ann. of Math. (2) $\mathbf{7 8}$ (1963), 365-416

[MePo1] P. Menal-Ferrer, J. Porti, Twisted cohomology for hyperbolic three manifolds, Preprint 2010, arXiv: 1001.2242

[MePo2] P. Menal-Ferrer, J. Porti, Higher dimensional Reidemeister torsion invariants for cusped hyperbolic 3-manifolds, Preprint 2011, arXiv:1110.3718

[Mü1] W. Müller, Analytic torsion and R-torsion of Riemannian manifolds, Adv. in Math. 28 (1978), 233-305

[Mü2] W. Müller, Analytic torsion and R-torsion for unimodular representations, J. Amer. Math. Soc. 6 (1993), 721-753

[Mü3] W. Müller, The asymptotics of the Ray-Singer analytic torsion of hyperbolic 3 manifolds, Preprint 2010, arXiv:1003.5168, to appear in: Metric and Differential Geometry, a volume in honor of Jeff Cheeger for his 65th birthday, Progress in Mathematics, Birkhäuser, 2012

[MP1] W. Müller, J. Pfaff, The asymptotics of the Ray-Singer analytic torsion for compact hyperbolic manifolds, Preprint 2011, arXiv:1108.2454

[MP2] W. Müller, J. Pfaff, Analytic torsion of complete hyperbolic manifolds of finite volume, Preprint 2011, arXiv:1110.4065

[Pa] J. Park, Analytic torsion and Ruelle zeta functions for hyperbolic manifolds with cusps, J. Funct. An. 257 (2009), no. 6, 1713-1758

[Pf] J. Pfaff, Selberg zeta functions on odd-dimensional hyperbolic manifolds of finite volume, Preprint 2012, arXiv:1205.1754

[Wo] A. Wotzke, Die Ruellesche Zetafunktion und die analytische Torsion hyperbolischer Mannigfaltigkeiten, Bonner Mathematische Schriften 389 (2008)

Universität Bonn, Mathematisches Institut, Endenicher Alle 60, D - 53115 Bonn, GerMANY

E-mail address: pfaff@math.uni-bonn.de 